\title{
Dual flexible rotor system with active magnetic bearings for unbalance and coupling misalignment faults analysis
}

\author{
SAMPATH KUMAR KUPPA and MOHIT LAL*(1) \\ Department of Industrial Design, National Institute of Technology Rourkela, Rourkela 769008, India \\ e-mail: 515id1003@nitrkl.ac.in; lalm@nitrkl.ac.in
}

MS received 5 December 2018; revised 5 May 2019; accepted 20 June 2019

\begin{abstract}
Rotating machines are the backbone of the present industrial world. Early fault detection and conditioning of these machines are primary concern of the researchers associated in this field. There are various faults (assembly error, coupling misalignment, looseness, imbalance, rotor crack, etc.) that cause malfunction of rotating machinery. Imbalance is one of the oldest problem and still challenging to perfectly balance the rotor. Imbalance leads to another inherent fault, i.e., coupling misalignment, especially in dual rotor or rotor train system. Imbalance and misalignment cause excessive vibration in the system that tends to shatter failure of the critical components of rotating machinery. In this article, active magnetic bearings (AMBs) are utilized to suppress the excessive vibration generated due to imbalance and misalignment. To regulate the controlling current of AMB a proportional integral derivative (PID) feedback controller is employed. A quantification technique is suggested to evaluate the tuned AMB characteristics along with imbalance and coupling misalignment dynamic parameters. A finite element method (FEM) modelling with high-frequency reduction scheme is utilized to acquire reduced system equations of motion. There are two advantages of employing condensation scheme, first, it reduces the number of sensors required and second, only linear (practically measurable) degrees of freedom are present in equations of motion derived. A SIMULINK ${ }^{\mathrm{TM}}$ code is prepared to solve a reduced linear differential equation. The time series feedback signals (current and displacement) obtained are transformed into a frequency series utilizing Fast Fourier Transformation (FFT) and utilized in developed algorithm. To establish the accuracy and effectiveness of the methodology, the estimated parameters are evaluated under two different frequency bands against measurement and modelling error (5\% variation in mass of the disc and bearing characteristic parameters).
\end{abstract}

Keywords. Finite element method; active magnetic bearings (AMBs); dual rotor; misalignment; full spectrum; high-frequency reduction.

\section{Introduction}

Rotor misalignment at coupling location is the second most common flaw after inherent imbalance in rotating machinery. The outcome of severe vibration in the rotating machinery often leads to sudden failure, critical damages to the components and huge monetary loss to the industries. Characteristic parameters related to the coupling misalignment as well as inherent unbalance must be identified at an initial phase. At present, active magnetic bearings (AMBs) are rapidly employed in industries for active controlling and stabilization of the system under different fault condition states [1-3].

Schweitzer and Maslen [1] proposed different control techniques for rigid as well as flexible rotor system and estimated fault parameters. An identification methodology to detect and quantify faults of fully levitated flexible rotor AMB system is developed by [2]. They performed a

*For correspondence

Published online: 26 July 2019 numerical experiment to test the methodology against measurement noise and modelling error. The dynamic behavior and stability of an asymmetric flexible rotor supported by AMB are analyzed by [3]. Floquet's theory was used to investigate the system stability.

At an early era of online condition monitoring, an algorithm was developed with the help of single magnetic actuator to estimate the system characteristics and to reduce excessive vibration generated on rotor system supported by conventional bearings [4]. They proposed an algorithm to determine the optimal control force to reduce the synchronous vibration of the system. Due to the advances in microprocessing controllers, application of AMB was reviewed to investigate the usage of magnetic bearings in gas turbine engines for online condition monitoring [5]. In article [6] nonlinear characteristics of AMB-rotor system is accessed using multiple scales technique. The nonlinear vibrational responses of the rotor system are varied with the variation of system parameters and based on system 
resonance information they estimated system stability. Authors of [7] developed an algorithm based on the multipoint method to measure the forces in AMB integrated rotor system. The accuracy of the force measurement in the system is improved by system identification approach. The application of AMB extended and used in machine tool industries [8]. They developed control system design for tool industries using AMB for its stabilization and better performance. In pair of research articles published in 2011 and 2013, coupling misalignment received first attention in AMB integrated rotor systems [9] and [10]. They used Newmark time integration method to derive the non-linear equation of motions (EOMs) of the system and determine the transient response of the system. The dynamic behavior of the misaligned rotor with $\mathrm{AMB}$ is predominant. To determine the transient response of the system, three radial AMBs with 4-pole, 6-pole and 8-pole are considered. A PID feedback controller mechanism is suggested to stabilize the fully suspended AMB system composed of 2-radial and 1-thrust active magnetic bearing [11]. They concluded the convergence error obtained from PID neural network is regulated by using Lyapunov function. A process is proposed to determine the equivalent elasticity of the AMB of multi-degrees of freedom system while considering the multi-frequency response data [12]. They suggested that the identification error can be reduced by selecting the relative phase of the lowest peak value in the multi-frequency excitation. The robustness and performance of the proposed method were checked against several measurement errors. AMB was used as an exciter to generate the force response data which were further used to develop an algorithm to evaluate the AMB dynamic parameters, both theoretically and experimentally [13]. A methodology was suggested that utilizes the unbalance response of the AMB integrated rotor to estimate the AMB closed-loop characteristic parameters [14]. The proposed methodology was tested for two different rotor model, i.e., rigid rotor model and flexible rotor model. The results concluded that the algorithm is robust for both models. An adaptive notch filter is proposed to suppress the synchronous vibration and increase the stability of an unbalanced rotorAMB system for high speed operations [15].

Recently, researchers have proposed an approach to quantitatively evaluate the characteristic parameters of a coupled rigid rotor comprises of the flexible bearing system supported by AMB [16]. They applied Lagrange's equation to develop linear EOMs of the system. An extension of [16] is presented by authors of [17] by considering flexible shaft system and applying a more practical solution approach, i.e., finite element method (FEM). The influence of bias current is studied to identify the center position of AMB [18]. They presented a bias current perturbation technique to determine the magnetic axis and measurement of current force; also, identified the influence of air gaps in the AMB system. A Timoshenko beam theory along with the finite element rotor model is considered to identify the AMB dynamic parameters [19]. The numerically simulated unbalance trajectory tested against experiments and found similar. An offset free predictive control method is presented to know the stability and control performance of the AMB in various applications [20]. The effectiveness of the method is validated with the help of tracking performance and estimated low-frequency disturbances of AMB system.

Authors of [21] described the nonlinear dynamic behavior of the AMB supported flexible rotor system. They used the Runge Kutta method to solve the EOM of the system. The effect of bearing force on the characteristic of dynamic system is investigated by power spectra analysis, dynamic trajectories and bifurcation diagrams. An active control technique is developed to know the stability of the rotor system [22]. Here, an integral actuator is used as a vibration control feedback. Article [23] discussed the control issues and dynamic characteristics of the AMB supported rotor system. The vibration levels in the rotor system are suppressed with the help of AMB. A re-levitation technique is developed to suppress the whirl motion in the AMB system [24]. Here PID is used as a controller in the AMB system to reduce the computational time. Authors of [25] developed an identification method based on two independent unbalance response datasets to identify the parameters (stiffness and damping) of a flexible rotor AMB system.

Based on the literature survey carried out it could be concluded that the application of AMB in misaligned rotor system is very limited. Misalignment is the second most common fault after unbalance that presents in the rotor system and needs special attention. In rotor train system modelling of misalignment is a real challenge. The present work is an extension of [17] for more realistic turbine generator model with the inclusion of damping and gyroscopic effect. In addition, the present work estimates coupling misalignment forces and moments along with AMB dynamic parameters. Moreover, to eliminate the rotational degrees of freedom the high-frequency reduction scheme is applied for the first time in AMB integrated rotor system. A quantification methodology is developed to quantify AMB characteristics along with imbalance and coupling misalignment characteristic parameters. Dual flexible shafts coupled with an elastic coupling, mounted over flexible bearings at ends are considered for numerical analysis. To demonstrate the developed methodology, a numerical experiment has been performed and the effectiveness has been established by verifying the methodology against modelling and measurement error. Also, the effectiveness of the algorithm is verified with repeatability analysis on estimated parameters at different frequency ranges or different modes of excitation.

\section{Model description}

This section explains the assumptions involved in modelling the turbogenerator system as a dual flexible rotor with AMB. 


\subsection{Assumptions and description of the model}

A system comprises of a dual flexible rotor with AMB as depicted in figure 1 , is taken into account to mimic and online health monitoring of the real turbogenerator system. A known beam theory (Euler Bernoulli) and FEM (figure 2) is utilized to describe the flexible shafts and to obtain elemental equations, respectively. With four stiffness $\left(k_{i j}^{B_{n}}\right)$ and four damping $\left(c_{i j}^{B_{n}}\right)$ bearings are defined. The coupling is modelled with stiffness $\left(k_{i j}^{C}, k_{\varphi x}^{C}\right.$ and $\left.k_{\varphi y}^{C}\right)$ and damping $\left(c_{i j}^{C}\right)$ parameters. Along with linear stiffness, two additional direct angular stiffness parameters are used in coupling to accommodate parallel as well as angular misalignment, respectively. Superscripts ' $B$ ' and ' $C$ ' stands for bearing and coupling, respectively.

\subsection{Modelling of the $A M B$}

Active magnetic bearings are used to suppress the excessive vibration generated due to various faults (unbalance and misalignment) present in the system. AMB controlling force can be considered in a manner such that the closed loop of the system behaves same as the mechanical spring damper system. The quality of the closed loop depends on eigenvalues and stiffness (displacement and current) of the AMB. Eight-Pole radial electromagnets are considered to define the characteristic parameters of AMB [1] and [2]. The AMB force could be given as,

$$
\boldsymbol{F}_{A M B}=-\boldsymbol{k}_{s} \boldsymbol{u}+\boldsymbol{k}_{i} \boldsymbol{i}_{c}
$$

with

$$
i_{c}=k_{P} u+k_{D} \dot{u}+k_{I} \int u
$$

where $k_{P}, k_{D}, k_{I}$ and $i_{c}$ are gain parameters of controller namely, proportional gain, derivative gain, integral gain and controlling current, respectively. $k_{s}$ and $k_{i}$ are displacement and current stiffness of AMB, respectively. Here, $u$ is the displacement vector (translational displacement at AMB location) with respect to time. The PID controller is used to correlate the AMB coil current with the system displacement. Once the AMB force is derived in terms of the system displacements, this can be appropriately incorporated into the system's EOM to develop identification algorithm.

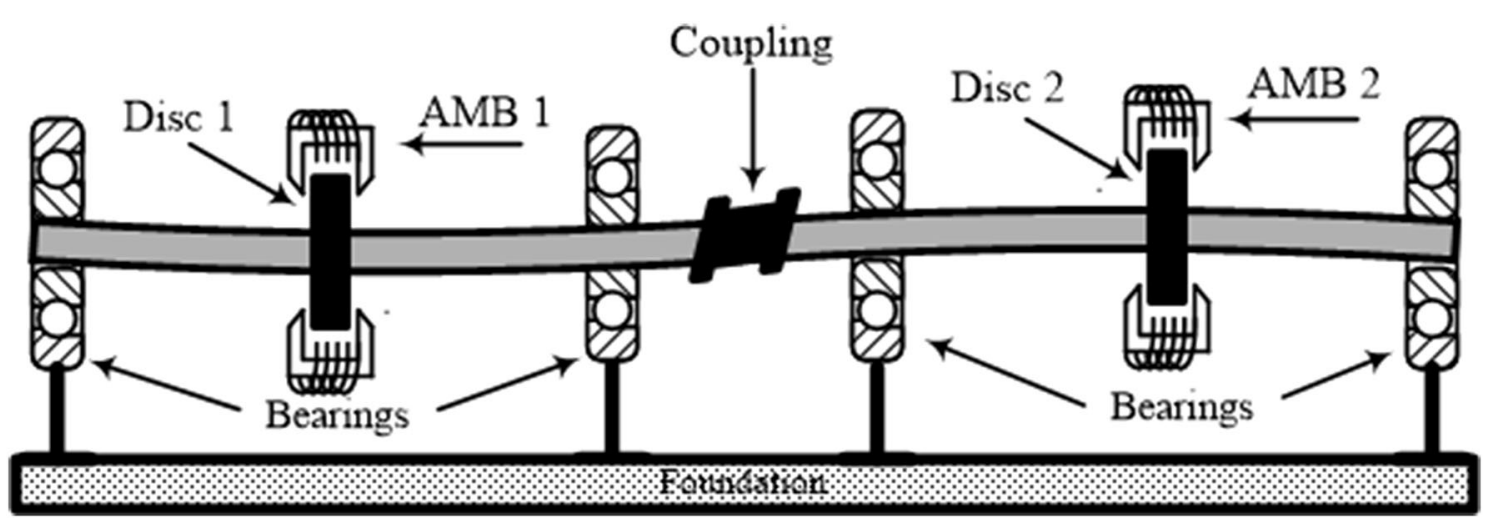

Figure 1. Dual flexible rotor with active magnetic bearings.

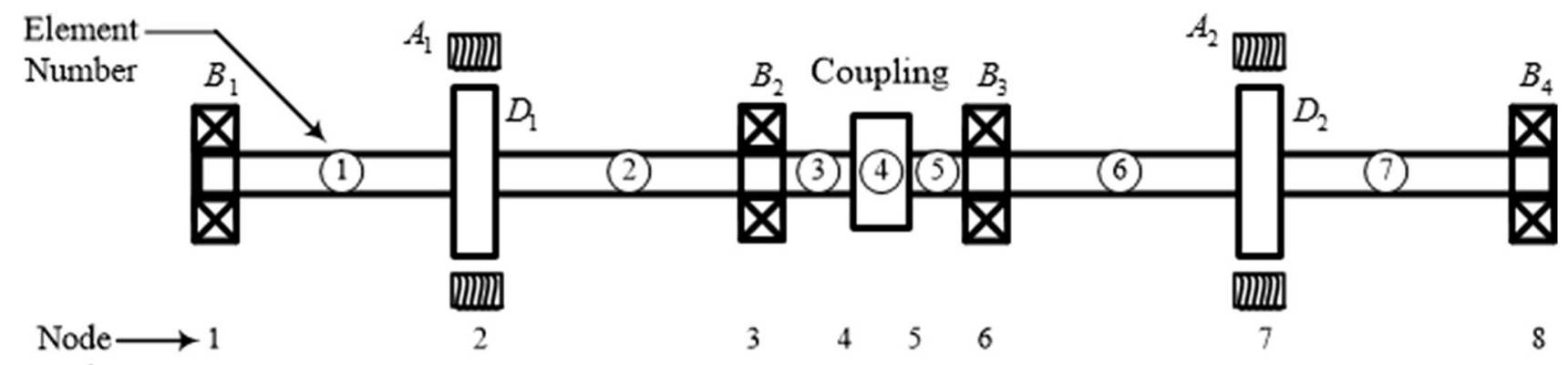

Figure 2. Elemental representation of dual rotor system. 


\subsection{Modelling of the rotor substructures}

Flexible shafts, rigid disc, flexible bearings and flexible coupling may define as the substructures of the rotor. The modelling and formulation of these substructures along with residual unbalance are well described in the literature [9], [26] and [27]. The assembled EOM due to unbalance force and AMB force may be expressed as

$$
\boldsymbol{M} \boldsymbol{X}+(\boldsymbol{C}-\omega \boldsymbol{G}) \dot{\boldsymbol{X}}+\boldsymbol{K} \boldsymbol{X}=\boldsymbol{F}_{\boldsymbol{u}}(\boldsymbol{t})-\boldsymbol{F}_{\boldsymbol{A M B}}
$$

where $\boldsymbol{X}_{32 \times 1}=\left\{x_{1}, y_{1}, \varphi_{y 1}, \varphi_{x 1} \ldots \mathrm{u} x_{8}, y_{8}, \varphi_{y 8}, \varphi_{x 8}\right\}^{T}$ represents the global displacement vector for global mass $\left(\boldsymbol{M}=\boldsymbol{M}_{\boldsymbol{S}+\boldsymbol{D}}\right)$, global damping $\left(\boldsymbol{C}=\boldsymbol{C}_{\boldsymbol{B}+\boldsymbol{C}+\boldsymbol{S}}\right)$, global gyroscopic $\left(\boldsymbol{G}=\boldsymbol{G}_{\boldsymbol{D}}\right), \quad$ global stiffness $\left(\boldsymbol{K}=\boldsymbol{K}_{\boldsymbol{B}+\boldsymbol{C}+\boldsymbol{S}}\right)$ matrices. $\boldsymbol{F}_{\boldsymbol{u}}$ and $\boldsymbol{F}_{\boldsymbol{A M B}}$ stands for external forces i.e., unbalance and active magnetic bearing forces, respectively.

\section{Development of numerical signal}

The characteristic specification of dual rotor system and AMB is listed in table 1 [1], [17] and [28]. A forced response with phase plot is presented in figure 3 . The first six critical frequencies may be observed as, $\omega_{c r 1}=48 \mathrm{rad} /$ $\mathrm{s}, \quad \omega_{c r 2}=67 \mathrm{rad} / \mathrm{s}, \quad \omega_{c r 3}=200 \mathrm{rad} / \mathrm{s}, \quad \omega_{c r 4}=208 \mathrm{rad} / \mathrm{s}$, $\omega_{c r 5}=347 \mathrm{rad} / \mathrm{s}$ and $\omega_{c r 6}=364 \mathrm{rad} / \mathrm{s} \quad(1 \mathrm{rad} /$ $\mathrm{s}=1 / 2 \pi \mathrm{Hz}$.

Figure 4 represents the flow chart of the proposed identification methodology developed to estimate the characteristic fault parameters. To tune and stabilize the operation of AMB a PID controller is used. After tuning the parameters, Eq. (2), may rearrange to obtain a numerical signal in time domain as,

$$
\mathcal{X}=\boldsymbol{M}^{-1}\left(\boldsymbol{F}_{\boldsymbol{u}}(\boldsymbol{t})-\boldsymbol{F}_{\boldsymbol{A} M \boldsymbol{B}}-(\boldsymbol{C}-\omega \boldsymbol{G}) \dot{\boldsymbol{X}}-\boldsymbol{K} \boldsymbol{X}\right)
$$

Numerical signals (displacement and current) in time series may acquire with the help of Simulink ${ }^{\mathrm{TM}}$ model (figure 5) and Eq. (3). The explicit Simulink model for complex unbalance force $\left(\boldsymbol{F}_{\boldsymbol{u}}=\overline{\boldsymbol{F}}_{\boldsymbol{u}} \boldsymbol{e}^{\mathrm{j} \omega \mathrm{t}}\right)$ is shown in figure 6. A sample time-series signal acquired at one of the running frequency i.e., $51 \mathrm{~Hz}$ is presented in figure 7. After that orbit plot is drawn for displacement and current signals and illustrated in figure 8. Orbit plot is developed to check the stability of the system. Single loop uniform orbit shows stable system. As the proposed algorithm requires the

Table 1. Specifications of dual rotor system and AMB.

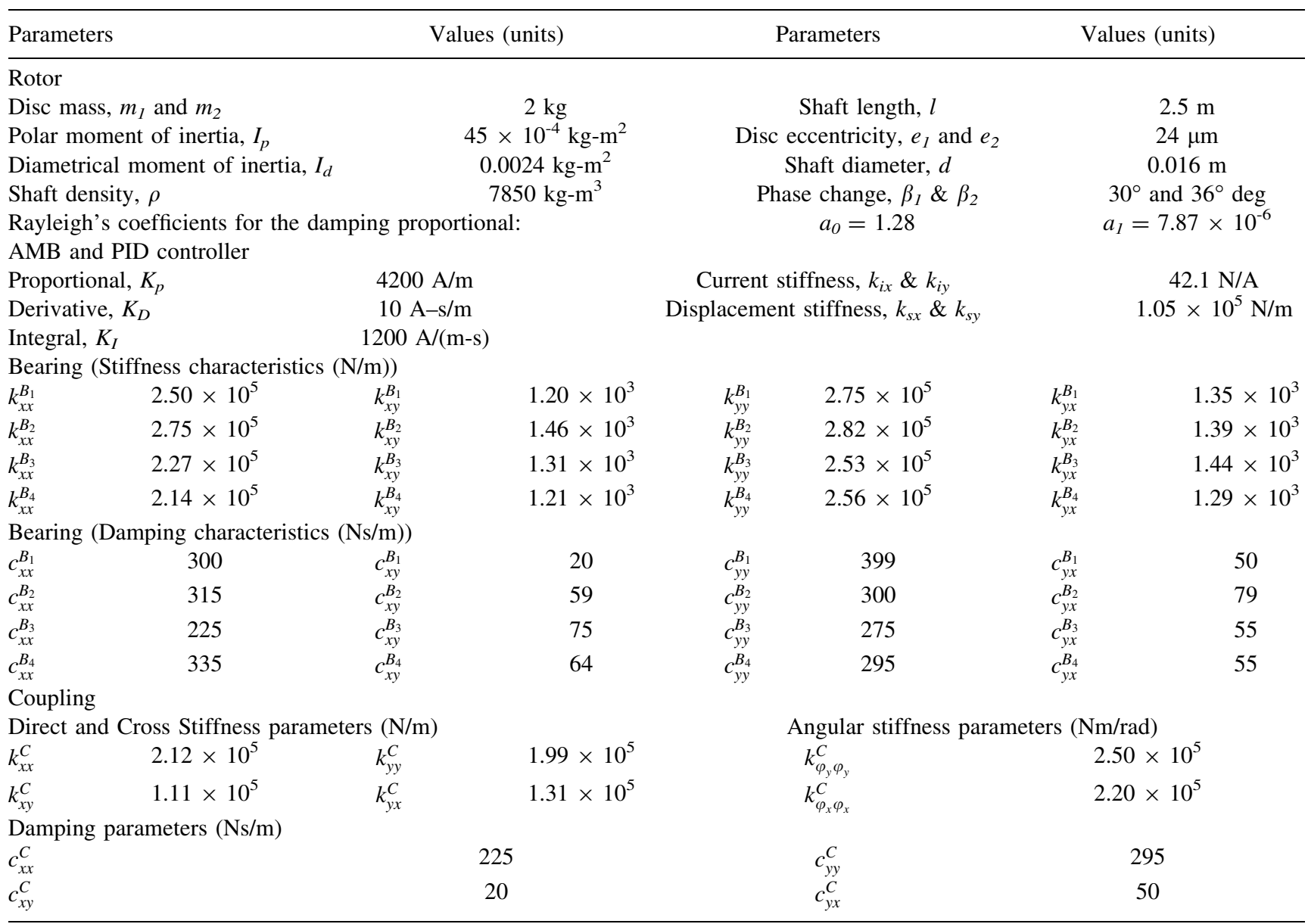




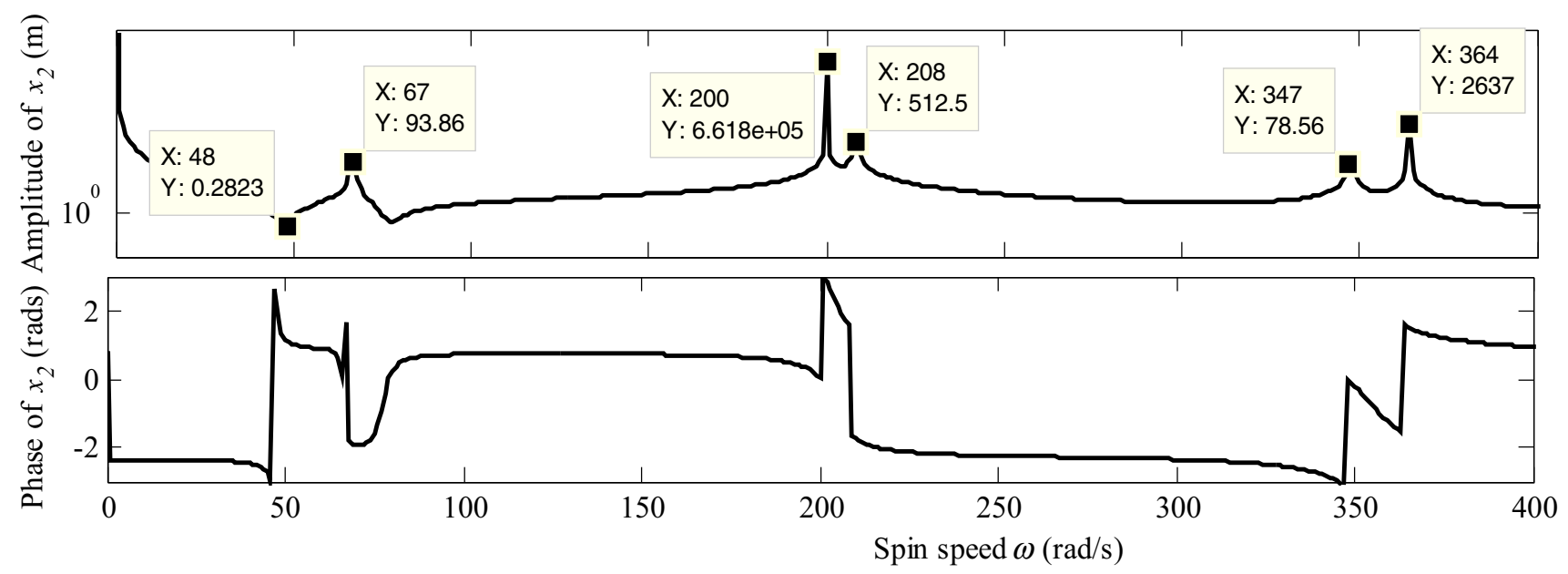

Figure 3. Forced response and phase plot with spin speed.

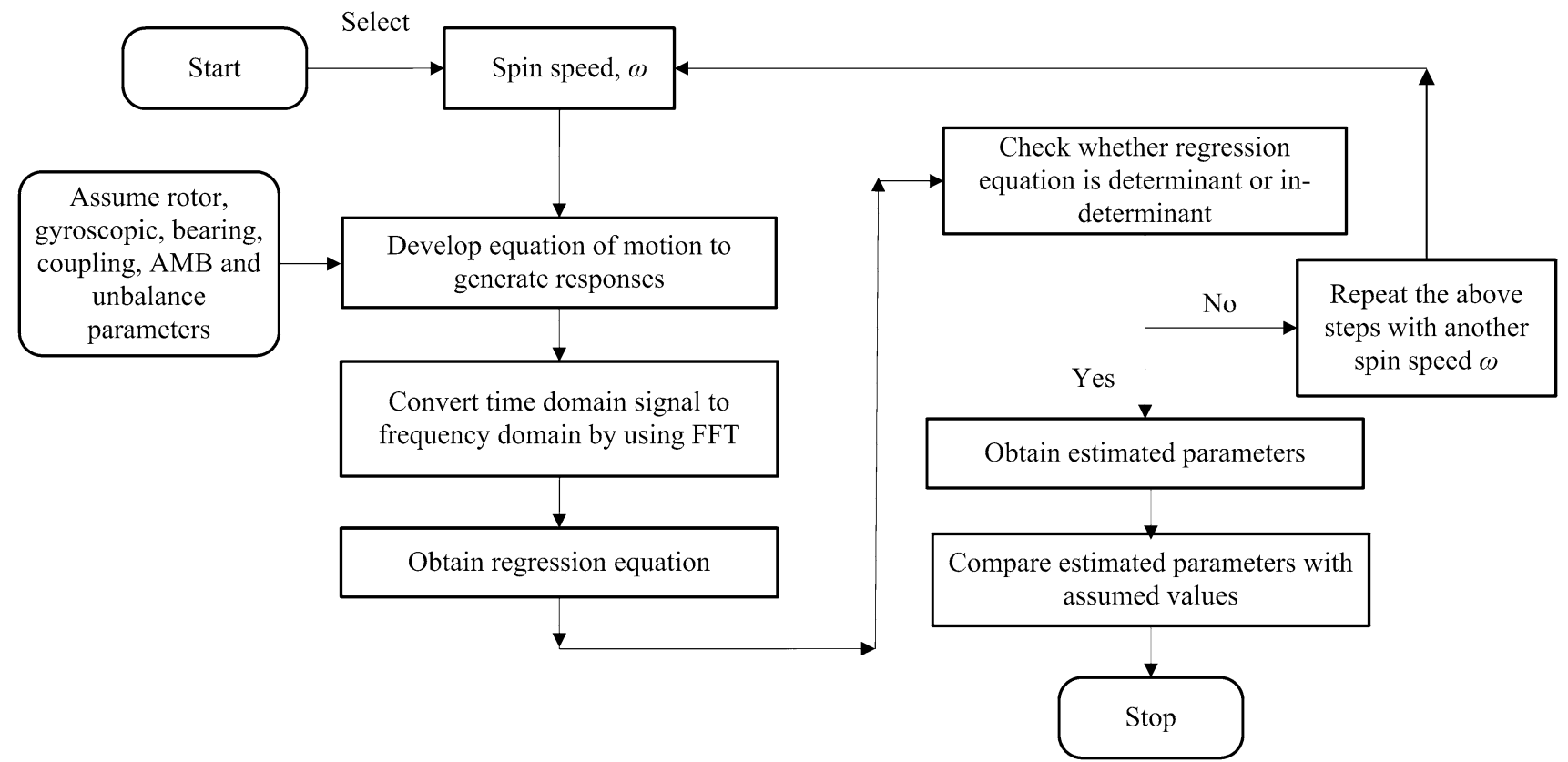

Figure 4. Flow chart of the identification algorithm.

system's information in frequency series the obtained signal is converted into the desired form (frequency series) using (FFT) approach and shown in figure 9. In figure 9, the characteristics for a peak at $1 \mathrm{~Hz}$ is mass of system whereas, peaks at $F_{r}+1(52 \mathrm{~Hz})$ and $-F_{r}(51 \mathrm{~Hz})$ shows the system running frequency in positive and negative abscissa, respectively. Same procedure is followed to obtain the signals at different operating speeds which are selected based on the system's critical frequencies. The splitting of natural frequency takes place due to large misalignment that leads to the influence of backward whirl in the system [29] and [30]. To consider the effect of forward and backward whirl, full spectrum plot is used. Also, to incorporate the effect of different modes of excitation, system information up to sixth critical frequencies are used to develop the estimation algorithm.

\section{Methodology development using dynamic reduction [31]}

Upon substitution, displacement, current and force as, $\boldsymbol{X}=\overline{\boldsymbol{X}} \boldsymbol{e}^{\mathrm{j} \omega \mathrm{t}}, \boldsymbol{I}=\overline{\boldsymbol{I}} \boldsymbol{e}^{\mathrm{j} \omega \mathrm{t}}$ and $\boldsymbol{F}_{\boldsymbol{u}}=\overline{\boldsymbol{F}}_{\boldsymbol{u}} \boldsymbol{e}^{\mathrm{j} \omega \mathrm{t}}$, respectively. Equation (2), in frequency domain may express as, 


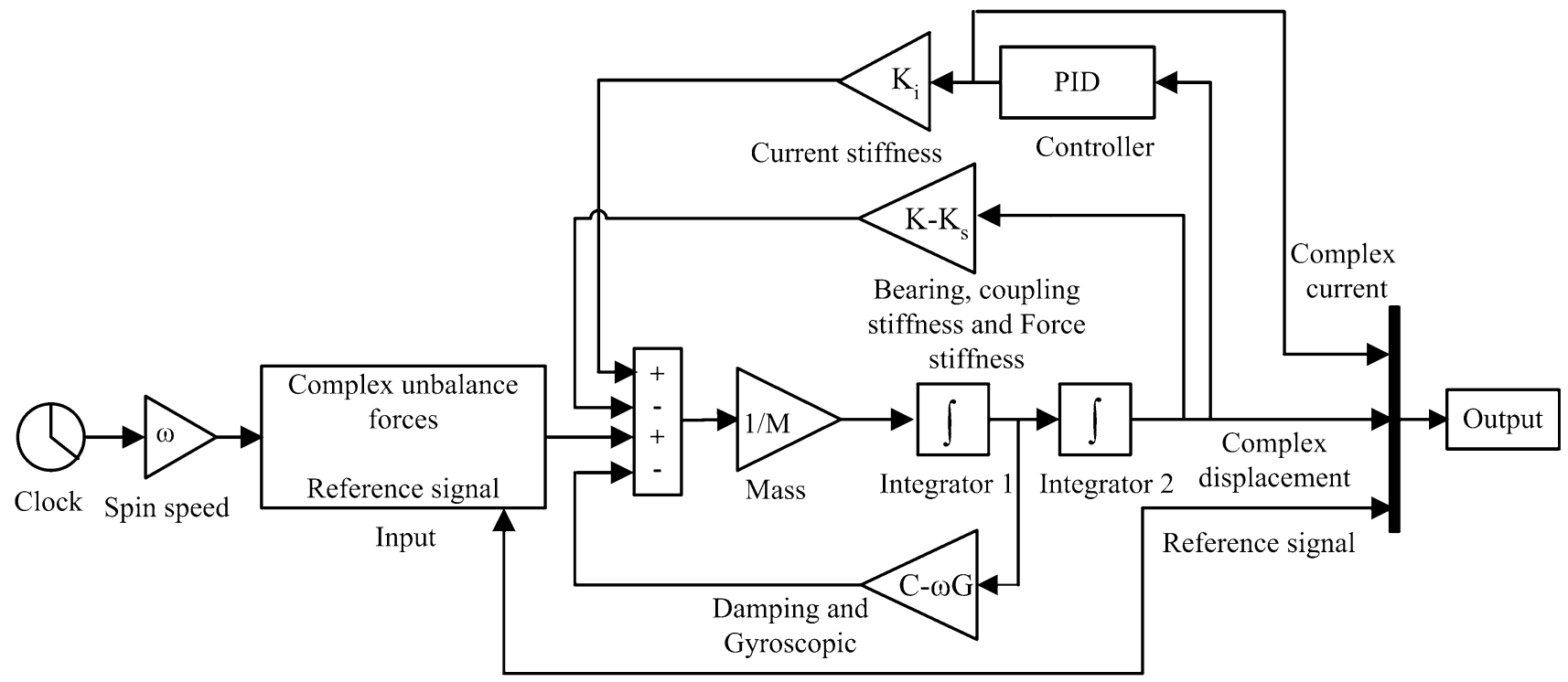

Figure 5. Representative Simulink model for dual rotor system with AMB.

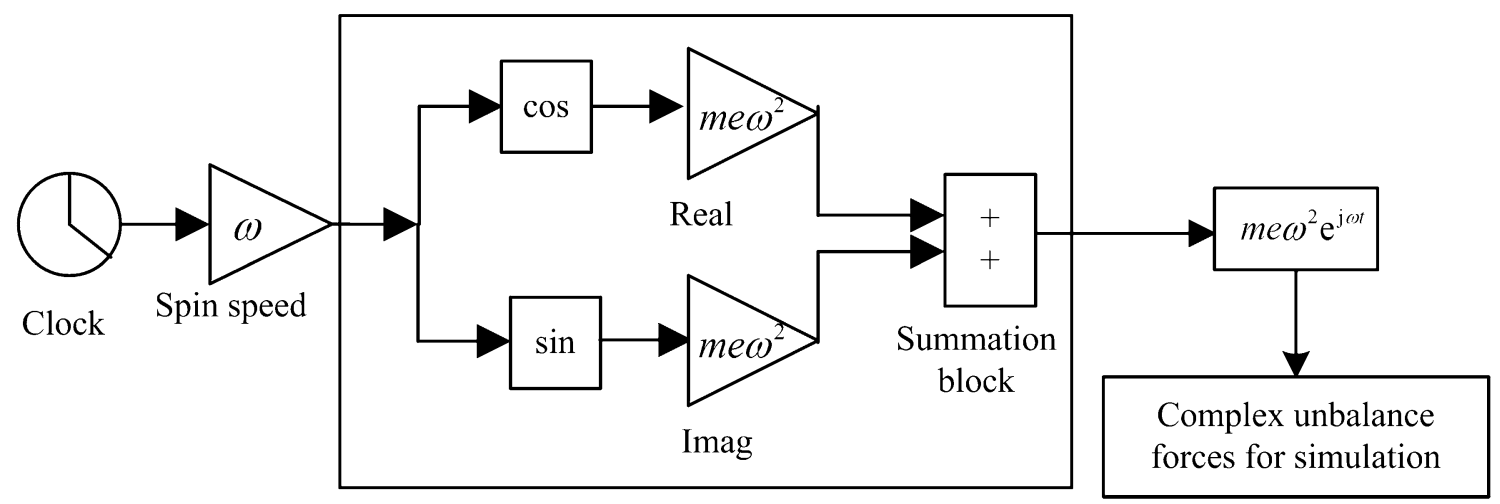

Figure 6. Explicit Simulink model for complex unbalance.

$$
\left[-\omega^{2} \boldsymbol{M}+\boldsymbol{j} \omega(\boldsymbol{C}-\omega \boldsymbol{G})+\boldsymbol{K}\right] \overline{\boldsymbol{X}}=\overline{\boldsymbol{F}}_{\boldsymbol{u}}-\overline{\boldsymbol{F}}_{\boldsymbol{A M B}}
$$

Due to the inadequate availability of a required number of sensors in the real-time situation, it is nearly impossible to access all the DOFs of the model. Also, it is highly unfeasible to measure the rotational DOFs. To encounter this limitation, a dynamic condensation scheme has been imposed in Eq. (4). To obtain reduced EOMs, Eq. (4) may partition into master and slave DOFs as,

$$
\begin{aligned}
& \left(-\omega^{2}\left[\begin{array}{ll}
\boldsymbol{M}_{\boldsymbol{m} m} & \boldsymbol{M}_{m s} \\
\boldsymbol{M}_{s m} & \boldsymbol{M}_{s s}
\end{array}\right]+\left[\begin{array}{cc}
\boldsymbol{K}_{\boldsymbol{m} m} & \boldsymbol{K}_{\boldsymbol{m}} \\
\boldsymbol{K}_{s m} & \boldsymbol{K}_{s s}
\end{array}\right]+\boldsymbol{j} \omega\right. \\
& \left.\left(\left[\begin{array}{ll}
\boldsymbol{C}_{\boldsymbol{m} m} & \boldsymbol{C}_{\boldsymbol{m} s} \\
\boldsymbol{C}_{\boldsymbol{s} \boldsymbol{m}} & \boldsymbol{C}_{s s}
\end{array}\right]-\omega\left[\begin{array}{ll}
\boldsymbol{G}_{\boldsymbol{m} m} & \boldsymbol{G}_{\boldsymbol{m}} \\
\boldsymbol{G}_{s m} & \boldsymbol{G}_{s s}
\end{array}\right]\right)\right)\left\{\begin{array}{c}
\overline{\boldsymbol{X}}_{\boldsymbol{m}} \\
\overline{\boldsymbol{X}}_{\boldsymbol{s}}
\end{array}\right\}=\left\{\begin{array}{c}
\overline{\boldsymbol{F}}_{\boldsymbol{m}} \\
\overline{\boldsymbol{F}}_{\boldsymbol{s}}
\end{array}\right\}
\end{aligned}
$$

The linear DOFs at bearing location and both (linear and angular) DOFs at coupling location are considered as masters DOFs, apart from these all other DOFs are considered as slave DOFs. Here, subscripts $m$ stands for master and $s$ stands for slave DOFs. After splitting and mathematical rearrangement Eq. (5), may be written as,

$$
\begin{aligned}
& -\omega^{2}\left(\boldsymbol{M}_{\boldsymbol{m} m} \overline{\boldsymbol{X}}_{\boldsymbol{m}}+\boldsymbol{M}_{\boldsymbol{m} s} \overline{\boldsymbol{X}}_{s}\right)+\boldsymbol{j} \omega\left(\boldsymbol{C}_{\boldsymbol{m} m}-\omega \boldsymbol{G}_{\boldsymbol{m} m}\right) \overline{\boldsymbol{X}}_{\boldsymbol{m}} \\
& \quad+\boldsymbol{j} \omega\left(\boldsymbol{C}_{\boldsymbol{m} s}-\omega \boldsymbol{G}_{\boldsymbol{m} s}\right) \overline{\boldsymbol{X}}_{s}+\boldsymbol{K}_{\boldsymbol{m} m} \overline{\boldsymbol{X}}_{\boldsymbol{m}}+\boldsymbol{K}_{\boldsymbol{m}} \overline{\boldsymbol{X}}_{s} \\
& \quad=\overline{\boldsymbol{F}}_{\boldsymbol{m}}
\end{aligned}
$$

and

$$
\begin{aligned}
& -\omega^{2}\left(\boldsymbol{M}_{s m} \overline{\boldsymbol{X}}_{\boldsymbol{m}}+\boldsymbol{M}_{s \overline{\boldsymbol{X}}} \overline{\boldsymbol{X}}_{s}\right)+\boldsymbol{j} \omega\left(\boldsymbol{C}_{s m}-\omega \boldsymbol{G}_{\boldsymbol{s}}\right) \overline{\boldsymbol{X}}_{\boldsymbol{m}} \\
& \quad+\boldsymbol{j} \omega\left(\boldsymbol{C}_{s s}-\omega \boldsymbol{G}_{s s}\right) \overline{\boldsymbol{X}}_{s}+\boldsymbol{K}_{s m} \overline{\boldsymbol{X}}_{\boldsymbol{m}}+\boldsymbol{K}_{s s} \overline{\boldsymbol{X}}_{s} \\
& \quad=\overline{\boldsymbol{F}}_{s}
\end{aligned}
$$



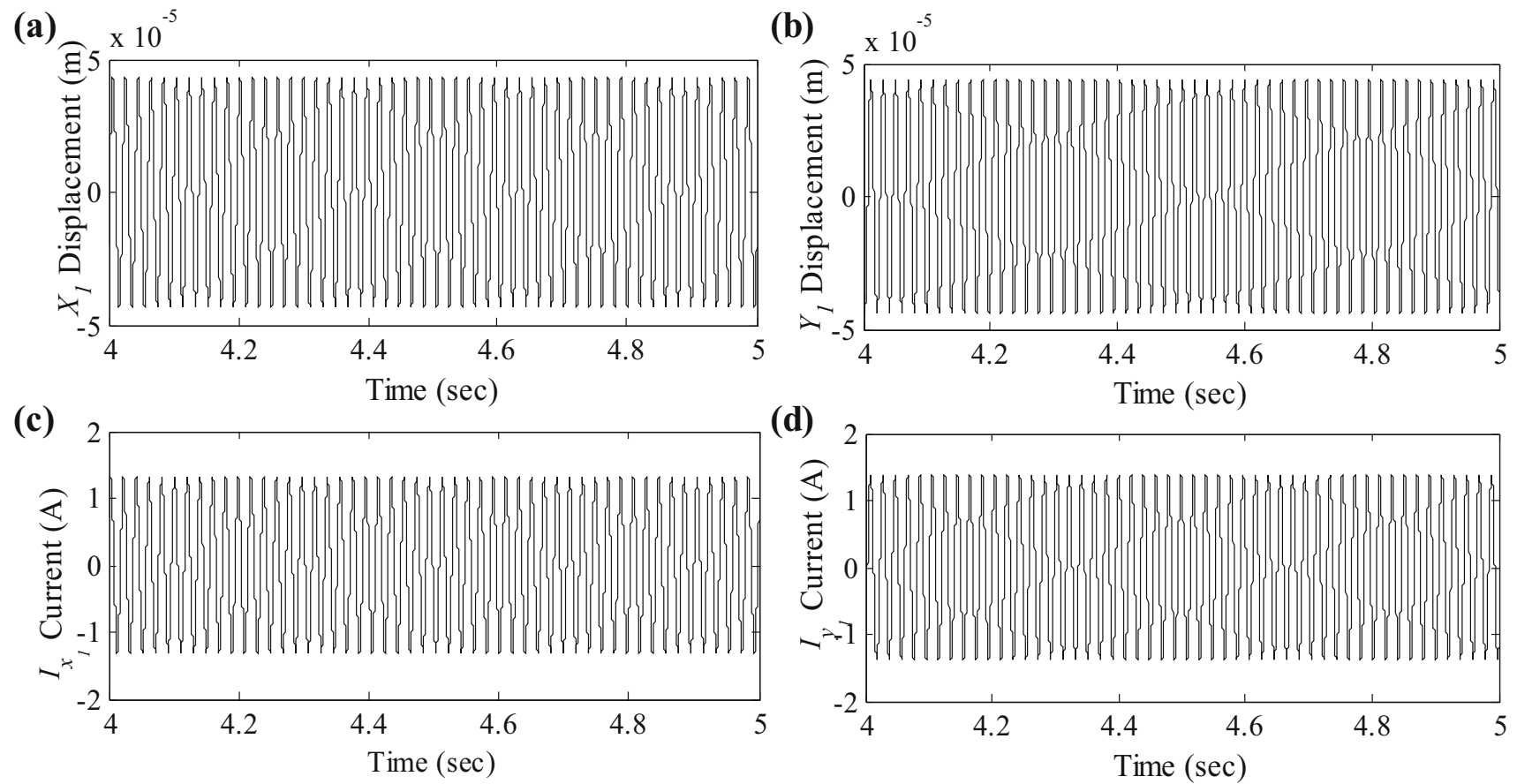

Figure 7. Numerical signal at $51 \mathrm{~Hz}$ (a) displacement $\mathrm{x}_{1},(\mathbf{b})$ displacement $\mathrm{y}_{1}$, (c) current $\mathrm{I}_{\mathrm{x} 1}$ and (d) current $\mathrm{I}_{\mathrm{y} 1}$.
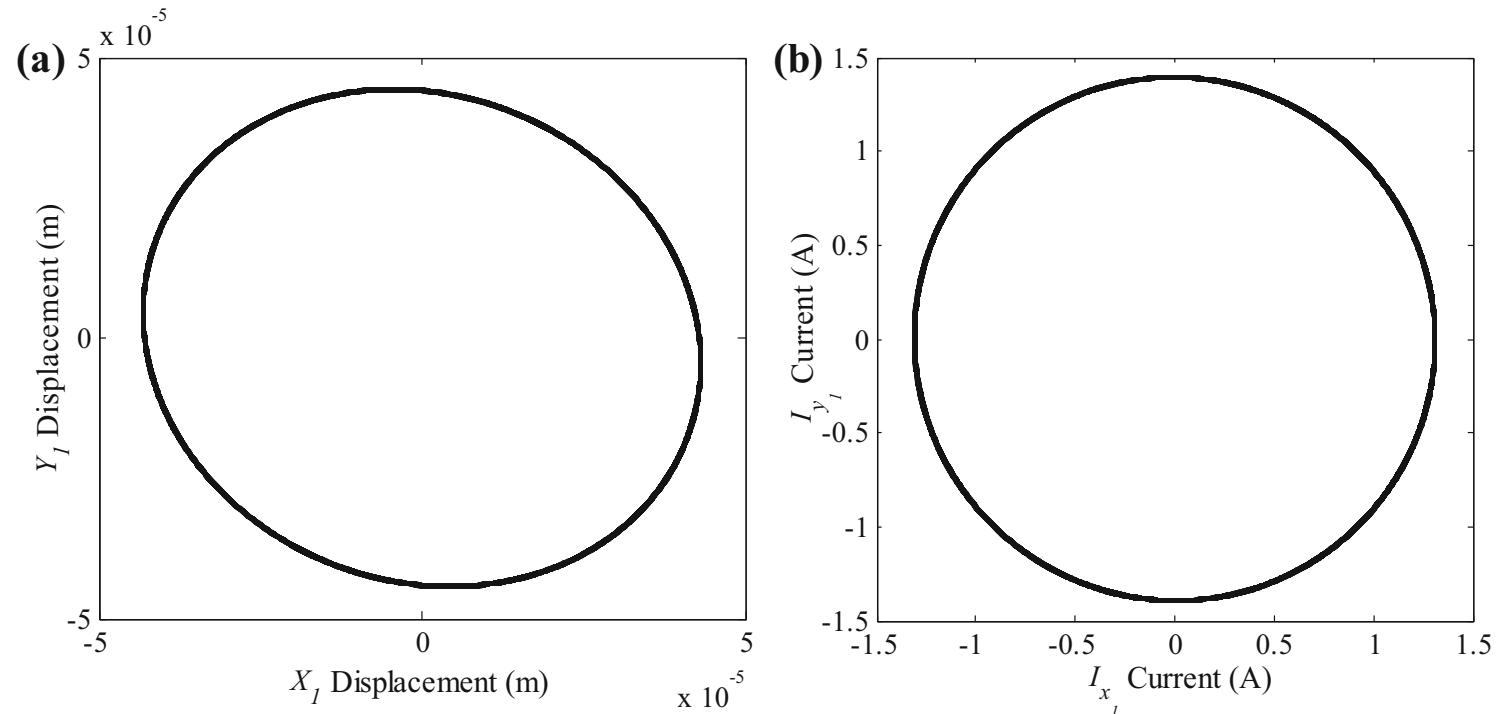

Figure 8. Orbit plot at $51 \mathrm{~Hz}$ for (a) shaft centerline and (b) current.

The assumptions involved in dynamic condensation scheme is given by (Paz [32], Friswell and Mottershead [33]). According to the assumptions the first order time derivative parameters (i.e., damping and gyroscopic) and force can be neglected. By following the above assumptions Eq. (7) can be written as,

$$
-\omega^{2}\left(\boldsymbol{M}_{s m} \overline{\boldsymbol{X}}_{\boldsymbol{m}}+\boldsymbol{M}_{s \boldsymbol{s}} \overline{\boldsymbol{X}}_{\boldsymbol{s}}\right)+\boldsymbol{K}_{\boldsymbol{s} \boldsymbol{m}} \overline{\boldsymbol{X}}_{\boldsymbol{m}}+\boldsymbol{K}_{\boldsymbol{s} \boldsymbol{s}} \overline{\boldsymbol{X}}_{\boldsymbol{s}}=0
$$

Separating Eq. (8) results,

$$
-\omega^{2} \boldsymbol{M}_{\boldsymbol{s} m} \overline{\boldsymbol{X}}_{\boldsymbol{m}}-\omega^{2} \boldsymbol{M}_{\boldsymbol{s}} \overline{\boldsymbol{X}}_{\boldsymbol{s}}+\boldsymbol{K}_{\boldsymbol{s} \boldsymbol{m}} \overline{\boldsymbol{X}}_{\boldsymbol{m}}+\boldsymbol{K}_{\boldsymbol{s} \boldsymbol{s}} \overline{\boldsymbol{X}}_{\boldsymbol{s}}=0
$$

Segregating the coefficients of masters $\left(\left(\bar{X}_{m}\right)\right)$ and slave $\left(\bar{X}_{s}\right)$ dofs Eq. (9) can be written as,

$$
\overline{\boldsymbol{X}}_{\boldsymbol{m}}\left(\boldsymbol{K}_{\boldsymbol{s} \boldsymbol{m}}-\omega^{2} \boldsymbol{M}_{s \boldsymbol{m}}\right)+\overline{\boldsymbol{X}}_{\boldsymbol{s}}\left(\boldsymbol{K}_{s s}-\omega^{2} \boldsymbol{M}_{s s}\right)=0
$$

With mathematical rearrangement Eq. (10) can be written as,

$$
\overline{\boldsymbol{X}}_{\boldsymbol{s}}=-\left(\boldsymbol{K}_{\boldsymbol{s s}}-\omega^{2} \boldsymbol{M}_{\boldsymbol{s} \boldsymbol{s}}\right)^{-1}\left(\boldsymbol{K}_{\boldsymbol{s} \boldsymbol{m}}-\omega^{2} \boldsymbol{M}_{\boldsymbol{s} \boldsymbol{m}}\right) \overline{\boldsymbol{X}}_{\boldsymbol{m}}
$$


(a)

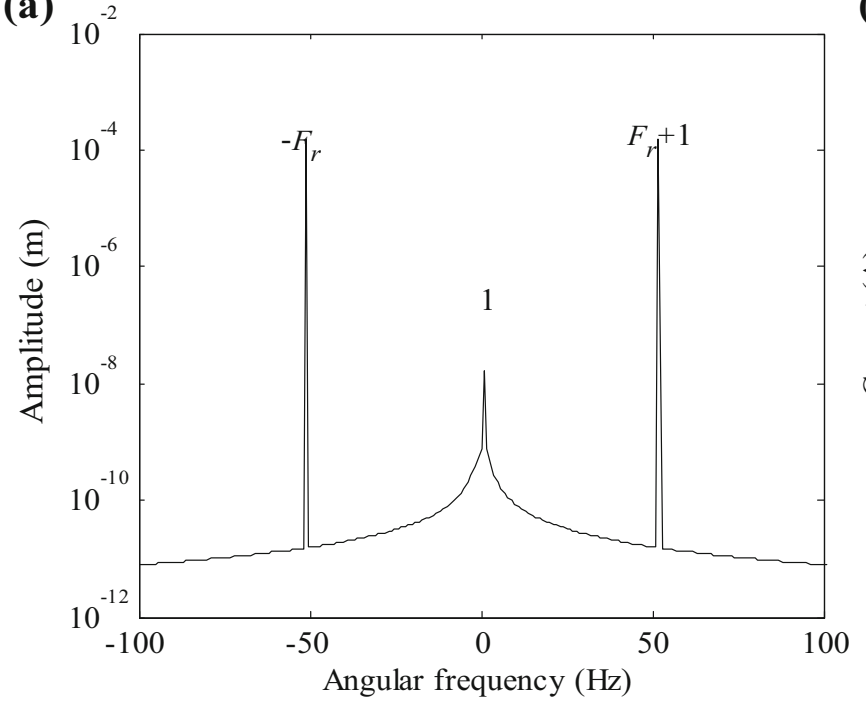

(b)

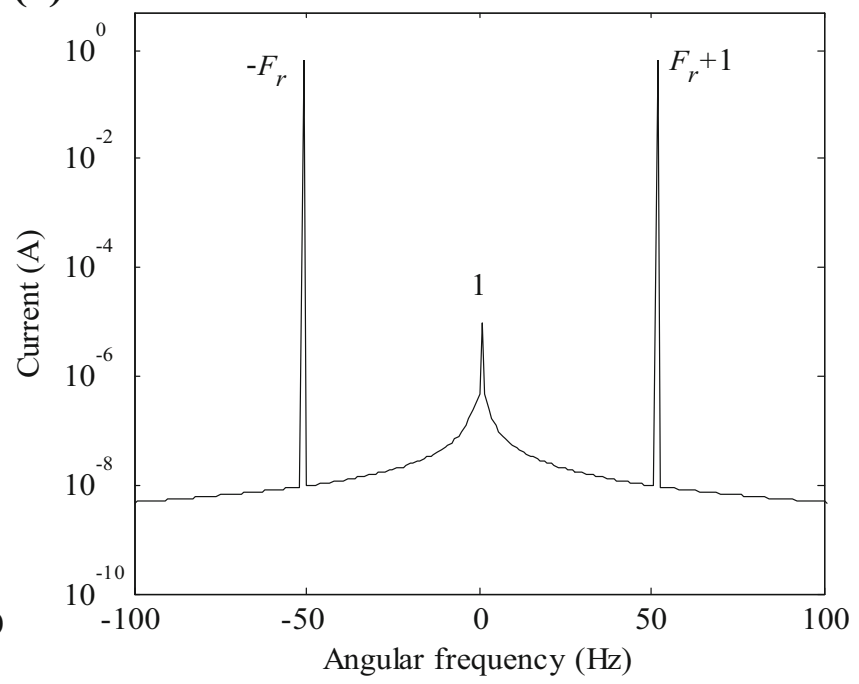

Figure 9. FFT plot at $51 \mathrm{~Hz}$ for (a) displacement amplitude and (b) current amplitude.

With identity matrix, state vector $\overline{\boldsymbol{X}}_{\boldsymbol{s}}$, may be written as,

$$
\bar{X}_{m}=\mathrm{I} \bar{X}_{m}
$$

where ' $\boldsymbol{I}$ ' represents the identity matrix. Equation (11) and (12), may combine as,

$$
\left\{\begin{array}{c}
\bar{X}_{m} \\
\bar{X}_{s}
\end{array}\right\}=T^{D} \bar{X}_{m}
$$

with

$$
\boldsymbol{T}^{\boldsymbol{D}}=\left[\frac{\mathrm{I}}{-\left(\boldsymbol{K}_{s s}-\omega^{2} \boldsymbol{M}_{s s}\right)^{-1}\left(\boldsymbol{K}_{s m}-\omega^{2} \boldsymbol{M}_{s m}\right)}\right]
$$

where $\boldsymbol{T}^{\boldsymbol{D}}$ is the transformation matrix. After putting Eq. (13), into Eq. (4), it may express as,

$$
\left[-\omega^{2} \boldsymbol{M}^{\boldsymbol{D}}+\boldsymbol{j} \omega\left(\boldsymbol{C}^{\boldsymbol{D}}-\omega \boldsymbol{G}^{\boldsymbol{D}}\right)+\boldsymbol{K}^{\boldsymbol{D}}\right] \overline{\boldsymbol{X}}_{\boldsymbol{m}}^{\boldsymbol{D}}=\overline{\boldsymbol{F}}_{u}^{\boldsymbol{D}}-\overline{\boldsymbol{F}}_{\boldsymbol{A M B}}^{\boldsymbol{D}}
$$

with,

$$
\begin{aligned}
& \boldsymbol{M}^{\boldsymbol{D}}=\left(\boldsymbol{T}^{\boldsymbol{D}}\right)^{\boldsymbol{T}} \boldsymbol{M} \boldsymbol{T}^{\boldsymbol{D}}, \boldsymbol{K}^{\boldsymbol{D}}=\left(\boldsymbol{T}^{\boldsymbol{D}}\right)^{\boldsymbol{T}} \boldsymbol{K} \boldsymbol{T}^{\boldsymbol{D}}, \boldsymbol{C}^{\boldsymbol{D}}=\left(\boldsymbol{T}^{\boldsymbol{D}}\right)^{\boldsymbol{T}} \\
& \boldsymbol{C} \boldsymbol{T}^{\boldsymbol{D}}, \boldsymbol{G}^{\boldsymbol{D}}=\left(\boldsymbol{T}^{\boldsymbol{D}}\right)^{\boldsymbol{T}} \boldsymbol{G} \boldsymbol{T}^{\boldsymbol{D}}, \\
& \overline{\boldsymbol{F}}_{\boldsymbol{U}}^{\boldsymbol{D}}=\left(\boldsymbol{T}^{\boldsymbol{D}}\right)^{\boldsymbol{T}} \overline{\boldsymbol{F}}_{\boldsymbol{u}}, \overline{\boldsymbol{F}}_{\boldsymbol{A M B}}^{\boldsymbol{D}}=\left(\boldsymbol{T}^{\boldsymbol{D}}\right)^{\boldsymbol{T}} \overline{\boldsymbol{F}}_{\boldsymbol{A M B}}, \text { and } \\
& \overline{\boldsymbol{X}}_{\boldsymbol{m}}^{\boldsymbol{D}}=\left\{\boldsymbol{x}_{1}, \boldsymbol{y}_{1}, \boldsymbol{x}_{2}, \boldsymbol{y}_{2}, \boldsymbol{x}_{3}, \boldsymbol{y}_{3}, \boldsymbol{x}_{4}, \varphi_{y_{4}}, \boldsymbol{y}_{4}, \varphi_{x_{4}}, \boldsymbol{x}_{5},\right. \\
& \left.\varphi_{y_{5}}, \boldsymbol{y}_{5}, \varphi_{x_{5}}, \boldsymbol{x}_{6}, \boldsymbol{y}_{6}, \boldsymbol{x}_{7}, \boldsymbol{y}_{7}, \boldsymbol{x}_{8}, \boldsymbol{y}_{8}\right\}^{\boldsymbol{T}}
\end{aligned}
$$

Here, $\left(x, y, \varphi_{y}\right.$ and $\left.\varphi_{x}\right)$ are the linear and angular displacements in $(x-z)$ and $(y-z)$ plane, respectively. From vector $\overline{\boldsymbol{X}}_{\boldsymbol{m}}^{\boldsymbol{D}}$ in Eq. (15), it may observe that dynamic reduction scheme has limitations, the associated DOFs corresponding to the parameters that need to be estimated cannot eliminate [28]. In the present research work, coupling and AMB characteristic parameters need to be evaluated. The corresponding linear and rotational DOFs at coupling locations as well as linear DOFs at AMB locations could not be eliminated from EOM with the help of dynamic reduction scheme. From Eq. (15), it could be seen that the reduced displacement vector $\bar{X}_{m}^{D}$ contains some immeasurable rotational DOFs and requires more (twenty) number of sensors for the present case. To overcome this limitation, along with dynamic reduction technique another reduction scheme, i.e., the high-frequency reduction has been applied in the subsequent section.

\section{Formulation of identification of algorithm using high-frequency reduction [28]}

The procedure followed from Eqs. (5) to (14), is applied on Eq. (15), to obtain EOM for high-frequency reduction scheme as,

$$
\left(-\omega^{2} \boldsymbol{M}^{\boldsymbol{H}}+\boldsymbol{j} \omega\left(\boldsymbol{C}^{\boldsymbol{H}}-\omega \boldsymbol{G}^{\boldsymbol{H}}\right)+\boldsymbol{K}^{\boldsymbol{H}}\right)\left\{\overline{\boldsymbol{X}}_{\boldsymbol{m}}^{\boldsymbol{H}}\right\}=\overline{\boldsymbol{F}}_{\boldsymbol{u}}^{\boldsymbol{H}}-\overline{\boldsymbol{F}}_{\boldsymbol{A M B}}^{\boldsymbol{H}}
$$

with

$$
\begin{aligned}
M^{H} & =\left(T^{H}\right)^{T} M^{D} T^{H}, K^{H}=\left(T^{H}\right)^{T} K^{D} T^{H}, C^{H} \\
& =\left(T^{H}\right)^{T} C^{D} T^{H}, G^{H}=\left(T^{H}\right)^{T} G^{D} T^{H}, \bar{F}_{u}^{H} \\
& =\left(T^{H}\right)^{T} \bar{F}_{u}^{D}, \\
\bar{F}_{A M B}^{H} & =\left(T^{H}\right)^{T} \bar{F}_{\mathrm{AMB}}^{\mathrm{D}} \text { and } \overline{\boldsymbol{X}}_{\boldsymbol{m}}^{\boldsymbol{H}} \\
& =\left\{\boldsymbol{x}_{1}, \boldsymbol{y}_{1}, \boldsymbol{x}_{3}, \boldsymbol{y}_{3}, \boldsymbol{x}_{6}, \boldsymbol{y}_{6}, \boldsymbol{x}_{8}, \boldsymbol{y}_{8}\right\}
\end{aligned}
$$

where 


$$
\boldsymbol{T}^{\boldsymbol{H}}=\left[\frac{\mathrm{I}}{-\left(\boldsymbol{M}_{s s}^{\boldsymbol{D}}\right)^{-1}\left(\boldsymbol{M}_{s m}^{\boldsymbol{D}}\right)}\right]
$$

Superscript $H$ represents high-frequency reduction. $T^{\boldsymbol{H}}$ is the transformation matrix. Vector $\bar{X}_{m}^{H}$ in Eq. (16), has the practically measurable quantity, i.e., only bearing transverse displacements. Now, Eq. (16) may be arranged to obtain standard regression from where the unknowns (AMB parameters (displacement stiffness and current stiffness), coupling parameters (stiffness and damping) and imbalances) on one side, and the knowns (global mass, damping (shaft and bearing), gyroscopic (disc) and stiffness (shaft and bearing)) on other side of the equation. The modified equation may represent as,

$$
\begin{aligned}
& \overline{\boldsymbol{F}}_{\boldsymbol{u}}^{\boldsymbol{H}}+\overline{\boldsymbol{F}}_{\boldsymbol{A M B}}^{\boldsymbol{H}}+\boldsymbol{K}^{\boldsymbol{H}(\boldsymbol{C})} \overline{\boldsymbol{X}}_{\boldsymbol{m}}^{\boldsymbol{H}}+\boldsymbol{j} \omega \boldsymbol{C}^{\boldsymbol{H}(\boldsymbol{C})} \overline{\boldsymbol{X}}_{\boldsymbol{m}}^{\boldsymbol{H}} \\
& =\left(\omega^{2} \boldsymbol{M}^{\boldsymbol{H}(\boldsymbol{D}+\boldsymbol{S})}-\boldsymbol{j} \omega\left(\boldsymbol{C}^{\boldsymbol{H}(\boldsymbol{B}+\boldsymbol{S})}-\omega \boldsymbol{G}^{\boldsymbol{H}(\boldsymbol{D})}\right)-\boldsymbol{K}^{\boldsymbol{H}(\boldsymbol{B}+\boldsymbol{R})}\right) \\
& \quad \overline{\boldsymbol{X}}_{\boldsymbol{m}}^{\boldsymbol{H}}
\end{aligned}
$$

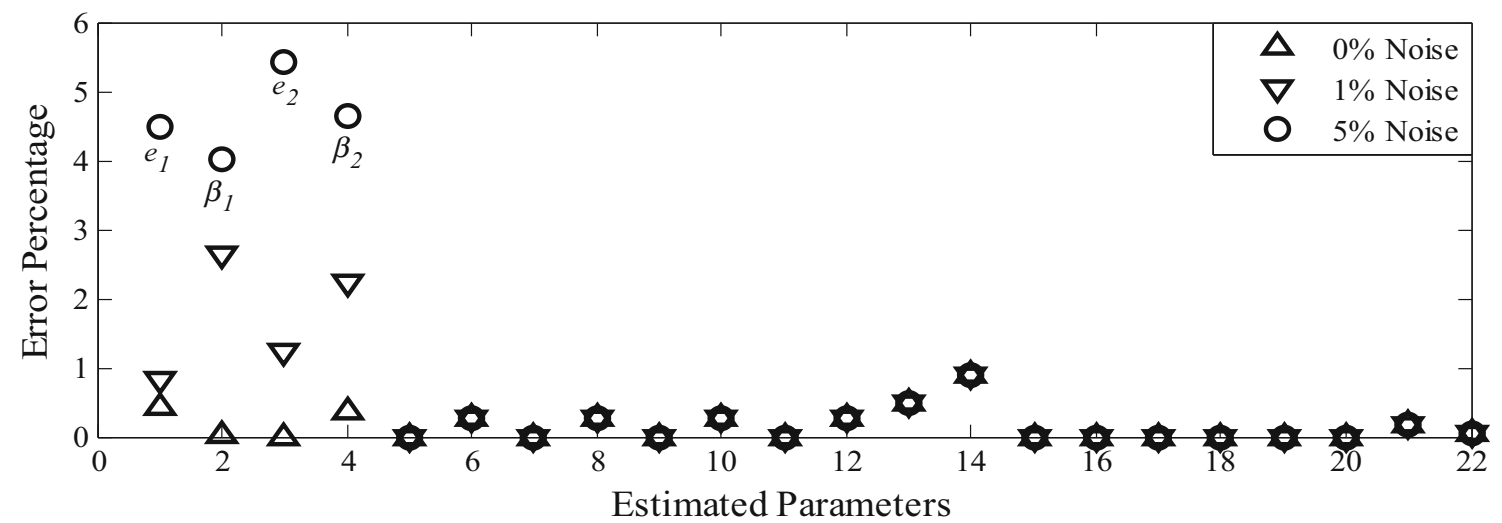

\begin{tabular}{|c|c|c|c|c|c|c|c|c|}
\hline \multirow[b]{3}{*}{ Sl.No. } & \multirow[b]{3}{*}{ Parameters (Units) } & \multirow[b]{3}{*}{ Assumed values } & \multicolumn{6}{|c|}{ Evaluated values } \\
\hline & & & \multicolumn{2}{|c|}{$0 \%$ Noise } & \multicolumn{2}{|c|}{$1 \%$ Noise } & \multicolumn{2}{|c|}{$5 \%$ Noise } \\
\hline & & & Values & (\% Error) & Values & (\% Error) & Values & (\% Error) \\
\hline 1 & $e_{1}(\mathrm{~mm})$ & $2.40 \times 10^{-4}$ & $2.39 \times 10^{-4}$ & 0.41 & $2.42 \times 10^{-4}$ & 0.83 & $2.51 \times 10^{-4}$ & 4.48 \\
\hline 2 & $\beta_{1}(\operatorname{deg})$ & $30^{\circ}$ & $30.01^{\circ}$ & 0.03 & $30.79^{\circ}$ & 2.63 & $31.21^{\circ}$ & 4.03 \\
\hline 3 & $e_{2}(\mathrm{~mm})$ & $2.40 \times 10^{-4}$ & $2.40 \times 10^{-4}$ & 0.00 & $2.43 \times 10^{-4}$ & 1.25 & $2.53 \times 10^{-4}$ & 5.41 \\
\hline 4 & $\beta_{2}(\operatorname{deg})$ & $36^{\circ}$ & $35.87^{\circ}$ & 0.36 & $36.81^{\circ}$ & 2.25 & $37.67^{\circ}$ & 4.63 \\
\hline 5 & $k_{s x_{1}}(\mathrm{~N} / \mathrm{m})$ & $1.05 \times 10^{5}$ & $1.05 \times 10^{5}$ & 0.00 & $1.05 \times 10^{5}$ & 0.00 & $1.05 \times 10^{5}$ & 0.00 \\
\hline 6 & $k_{i x_{1}}(\mathrm{~N} / \mathrm{A})$ & 42.00 & 41.88 & 0.28 & 41.88 & 0.28 & 41.88 & 0.28 \\
\hline 7 & $k_{s x_{2}}(\mathrm{~N} / \mathrm{m})$ & $1.05 \times 10^{5}$ & $1.05 \times 10^{5}$ & 0.00 & $1.05 \times 10^{5}$ & 0.00 & $1.05 \times 10^{5}$ & 0.00 \\
\hline 8 & $k_{i x_{2}}(\mathrm{~N} / \mathrm{A})$ & 42.00 & 41.88 & 0.28 & 41.88 & 0.28 & 41.88 & 0.28 \\
\hline 9 & $k_{s y_{1}}(\mathrm{~N} / \mathrm{m})$ & $1.05 \times 10^{5}$ & $1.05 \times 10^{5}$ & 0.00 & $1.05 \times 10^{5}$ & 0.00 & $1.05 \times 10^{5}$ & 0.00 \\
\hline 10 & $k_{i y_{1}}$ (N/A) & 42.00 & 41.88 & 0.28 & 41.88 & 0.28 & 41.88 & 0.28 \\
\hline 11 & $k_{s y_{2}}(\mathrm{~N} / \mathrm{m})$ & $1.05 \times 10^{5}$ & $1.05 \times 10^{5}$ & 0.00 & $1.05 \times 10^{5}$ & 0.00 & $1.05 \times 10^{5}$ & 0.00 \\
\hline 12 & $k_{i y_{2}}(\mathrm{~N} / \mathrm{A})$ & 42.0 & 41.88 & 0.28 & 41.88 & 0.28 & 41.88 & 0.28 \\
\hline 13 & $k_{x x}^{C}(\mathrm{~N} / \mathrm{m})$ & $2.12 \times 10^{5}$ & $2.11 \times 10^{5}$ & 0.47 & $2.11 \times 10^{5}$ & 0.47 & $2.11 \times 10^{5}$ & 0.47 \\
\hline 14 & $k_{x y}^{C}(\mathrm{~N} / \mathrm{m})$ & $1.11 \times 10^{5}$ & $1.10 \times 10^{5}$ & 0.90 & $1.10 \times 10^{5}$ & 0.90 & $1.10 \times 10^{5}$ & 0.90 \\
\hline 15 & $k_{y x}^{C}(\mathrm{~N} / \mathrm{m})$ & $1.31 \times 10^{5}$ & $1.31 \times 10^{5}$ & 0.00 & $1.31 \times 10^{5}$ & 0.00 & $1.31 \times 10^{5}$ & 0.00 \\
\hline 16 & $k_{y y}^{C}(\mathrm{~N} / \mathrm{m})$ & $1.99 \times 10^{5}$ & $1.99 \times 10^{5}$ & 0.00 & $1.99 \times 10^{5}$ & 0.00 & $1.99 \times 10^{5}$ & 0.00 \\
\hline 17 & $k_{\varphi_{x} \varphi_{x}}^{C}(\mathrm{Nm} / \mathrm{rad})$ & $2.20 \times 10^{5}$ & $2.20 \times 10^{5}$ & 0.00 & $2.20 \times 10^{5}$ & 0.00 & $2.20 \times 10^{5}$ & 0.00 \\
\hline 18 & $k_{\varphi_{y} \varphi_{y}}^{C}(\mathrm{Nm} / \mathrm{rad})$ & $2.50 \times 10^{5}$ & $2.50 \times 10^{5}$ & 0.00 & $2.50 \times 10^{5}$ & 0.00 & $2.50 \times 10^{5}$ & 0.00 \\
\hline 19 & $c_{x x}^{C}(\mathrm{Ns} / \mathrm{m})$ & 225 & 224.99 & 0.00 & 224.99 & 0.00 & 224.99 & 0.00 \\
\hline 20 & $c_{x y}^{C}(\mathrm{Ns} / \mathrm{m})$ & 20 & 20.00 & 0.00 & 20.00 & 0.00 & 20.00 & 0.00 \\
\hline 21 & $c_{y x}^{C}(\mathrm{Ns} / \mathrm{m})$ & 50 & 49.91 & 0.18 & 49.91 & 0.18 & 49.91 & 0.18 \\
\hline 22 & $c_{y y}^{C}(\mathrm{Ns} / \mathrm{m})$ & 295 & 294.84 & 0.05 & 294.84 & 0.05 & 294.84 & 0.05 \\
\hline
\end{tabular}

Figure 10. Effect of measurement noise on evaluated parameters for Case A.

Table 2. Evaluated parameters with percentage deviation against measurement noise for Case A. 
Table 3. Evaluated parameters with percentage deviation against measurement noise for Case B.

\begin{tabular}{|c|c|c|c|c|c|c|c|c|}
\hline \multirow[b]{3}{*}{ Sl.No. } & \multirow[b]{3}{*}{ Parameters (units) } & \multirow[b]{3}{*}{ Assumed values } & \multicolumn{6}{|c|}{ Evaluated values } \\
\hline & & & \multicolumn{2}{|c|}{$0 \%$ Noise } & \multicolumn{2}{|c|}{$1 \%$ Noise } & \multicolumn{2}{|c|}{$5 \%$ Noise } \\
\hline & & & Values & (\% Error) & Values & (\% Error) & Values & (\% Error) \\
\hline 1 & $e_{1}(\mathrm{~mm})$ & $2.40 \times 10^{-4}$ & $2.40 \times 10^{-4}$ & 0.00 & $2.42 \times 10^{-4}$ & 0.83 & $2.52 \times 10^{-4}$ & 5.00 \\
\hline 2 & $\beta_{1}(\operatorname{deg})$ & $30^{\circ}$ & $29.91^{\circ}$ & 0.30 & $30.58^{\circ}$ & 1.93 & 31.01 & 3.36 \\
\hline 3 & $e_{2}(\mathrm{~mm})$ & $2.40 \times 10^{-4}$ & $2.40 \times 10^{-4}$ & 0.00 & $2.44 \times 10^{-4}$ & 1.66 & $2.51 \times 10^{-4}$ & 4.58 \\
\hline 4 & $\beta_{2}(\operatorname{deg})$ & $36^{\circ}$ & $35.61^{\circ}$ & 1.08 & $36.73^{\circ}$ & 2.02 & 37.51 & 4.19 \\
\hline 5 & $k_{s x_{1}}(\mathrm{~N} / \mathrm{m})$ & $1.05 \times 10^{5}$ & $1.05 \times 10^{5}$ & 0.00 & $1.05 \times 10^{5}$ & 0.00 & $1.05 \times 10^{5}$ & 0.00 \\
\hline 6 & $k_{i x_{1}}(\mathrm{~N} / \mathrm{A})$ & 42.00 & 41.93 & 0.16 & 41.92 & 0.19 & 41.93 & 0.16 \\
\hline 7 & $k_{s x_{2}}(\mathrm{~N} / \mathrm{m})$ & $1.05 \times 10^{5}$ & $1.05 \times 10^{5}$ & 0.00 & $1.05 \times 10^{5}$ & 0.00 & $1.05 \times 10^{5}$ & 0.00 \\
\hline 8 & $k_{i x_{2}}(\mathrm{~N} / \mathrm{A})$ & 42.00 & 41.92 & 0.19 & 41.91 & 0.21 & 41.92 & 0.19 \\
\hline 9 & $k_{s y_{1}}(\mathrm{~N} / \mathrm{m})$ & $1.05 \times 10^{5}$ & $1.05 \times 10^{5}$ & 0.00 & $1.05 \times 10^{5}$ & 0.00 & $1.05 \times 10^{5}$ & 0.00 \\
\hline 10 & $k_{i y_{1}}$ (N/A) & 42.00 & 41.91 & 0.21 & 41.90 & 0.23 & 41.91 & 0.21 \\
\hline 11 & $k_{s y_{2}}(\mathrm{~N} / \mathrm{m})$ & $1.05 \times 10^{5}$ & $1.05 \times 10^{5}$ & 0.00 & $1.05 \times 10^{5}$ & 0.00 & $1.05 \times 10^{5}$ & 0.00 \\
\hline 12 & $k_{i y_{2}}(\mathrm{~N} / \mathrm{A})$ & $42 . .0$ & 41.90 & 0.23 & 41.90 & 0.23 & 41.90 & 0.23 \\
\hline 13 & $k_{x x}^{C}(\mathrm{~N} / \mathrm{m})$ & $2.12 \times 10^{5}$ & $2.11 \times 10^{5}$ & 0.47 & $2.118 \times 10^{5}$ & 0.47 & $2.11 \times 10^{5}$ & 0.47 \\
\hline 14 & $k_{x y}^{C x}(\mathrm{~N} / \mathrm{m})$ & $1.11 \times 10^{5}$ & $1.10 \times 10^{5}$ & 0.90 & $1.10 \times 10^{5}$ & 0.90 & $1.10 \times 10^{5}$ & 0.90 \\
\hline 15 & $k_{v x}^{C}(\mathrm{~N} / \mathrm{m})$ & $1.31 \times 10^{5}$ & $1.30 \times 10^{5}$ & 0.76 & $1.30 \times 10^{5}$ & 0.76 & $1.30 \times 10^{5}$ & 0.76 \\
\hline 16 & $k_{v y}^{C}(\mathrm{~N} / \mathrm{m})$ & $1.99 \times 10^{5}$ & $1.99 \times 10^{5}$ & 0.00 & $1.99 \times 10^{5}$ & 0.00 & $1.99 \times 10^{5}$ & 0.00 \\
\hline 17 & $k_{\varphi_{x} \varphi_{r}}^{C}(\mathrm{Nm} / \mathrm{rad})$ & $2.20 \times 10^{5}$ & $2.19 \times 10^{5}$ & 0.45 & $2.19 \times 10^{5}$ & 0.45 & $2.19 \times 10^{5}$ & 0.45 \\
\hline 18 & $k_{\varphi_{y} \varphi_{y}}^{C}(\mathrm{Nm} / \mathrm{rad})$ & $2.50 \times 10^{5}$ & $2.50 \times 10^{5}$ & 0.00 & $2.50 \times 10^{5}$ & 0.00 & $2.50 \times 10^{5}$ & 0.00 \\
\hline 19 & $c_{x x}^{C}(\mathrm{Ns} / \mathrm{m})$ & 225 & 224.99 & 0.00 & 224.99 & 0.00 & 224.99 & 0.00 \\
\hline 20 & $c_{x y}^{C}(\mathrm{Ns} / \mathrm{m})$ & 20 & 19.99 & 0.05 & 19.99 & 0.05 & 19.99 & 0.05 \\
\hline 21 & $c_{y x}^{C}(\mathrm{Ns} / \mathrm{m})$ & 50 & 50.08 & 0.16 & 50.08 & 0.16 & 50.08 & 0.16 \\
\hline 22 & $c_{y y}^{C}(\mathrm{Ns} / \mathrm{m})$ & 295 & 295.14 & 0.04 & 295.14 & 0.04 & 295.14 & 0.04 \\
\hline
\end{tabular}

Eq. (17), may be written as,

$$
\boldsymbol{A}\left(\omega_{i}\right) \chi=\boldsymbol{B}\left(\omega_{i}\right)
$$

with

$$
\begin{gathered}
\chi=\left\{\boldsymbol{e}_{1}, \beta_{1}, \boldsymbol{e}_{2}, \beta_{2}, \boldsymbol{k}_{s x_{1}}, \boldsymbol{k}_{i x_{1}}, \boldsymbol{k}_{s x_{2}}, \boldsymbol{k}_{i x_{2}}, \boldsymbol{k}_{s y_{1}}, \boldsymbol{k}_{i y_{1}}, \boldsymbol{k}_{s y_{2}}, \boldsymbol{k}_{i y_{2}},\right. \\
\left.\boldsymbol{k}_{x x}^{C}, \boldsymbol{k}_{x y}^{C}, \boldsymbol{k}_{y x}^{C}, \boldsymbol{k}_{y y}^{C}, \boldsymbol{k}_{\varphi_{x} \varphi_{x}}^{C}, \boldsymbol{k}_{\varphi_{y} \varphi_{y}}^{C}, \boldsymbol{c}_{x x}^{C}, c_{x y}^{C}, c_{y x}^{C}, c_{y y}^{C}\right\}^{\boldsymbol{T}}
\end{gathered}
$$

$\boldsymbol{A}$ and $\boldsymbol{B}$ are the regression matrices due to unknown and known parameters, respectively; details may be obtained from Appendix A. Matrix $\boldsymbol{A}$ contains coefficient contributions due to residual unbalances $\overline{\boldsymbol{F}}_{\boldsymbol{u}}^{\boldsymbol{H}}$, AMB force $\overline{\boldsymbol{F}}_{\boldsymbol{A M B}}^{\boldsymbol{H}}$, coupling stiffness $\boldsymbol{K}^{\boldsymbol{H}(\boldsymbol{C})}$ and coupling damping $\boldsymbol{C}^{\boldsymbol{H}(\boldsymbol{C})}$. Matrix $\boldsymbol{B}$ is coefficient contributions of known parameters contain mass (shaft and disc), $\boldsymbol{M}^{\boldsymbol{H}(\boldsymbol{D}+\boldsymbol{S})}$, stiffness (shaft and bearing), $\boldsymbol{K}^{\boldsymbol{H}(\boldsymbol{B}+\boldsymbol{S})}$, damping (shaft and bearing), $\boldsymbol{C}^{\boldsymbol{H}(\boldsymbol{B}+\boldsymbol{S})}$ and gyroscopic effect (disc), $\boldsymbol{G}^{\boldsymbol{H}(\boldsymbol{D})}$. Eq. (18) needs independently generated signal information to evaluate the characteristics kept in vector $\chi$. The two cases proposed to acquire numerical signals independently are,

Case A: Operating speeds in between the second and third critical frequency
Case B: Operating speeds in between the fourth and fifth critical frequency

In the subsequent section, based on the above two cases, operating speeds are selected to generate independent sets of response data. Also, to analyze the adaptability of the algorithm, the repeatability analysis is performed by estimating the parameters at different frequency ranges. The abscissa in figures 10-14, represent the parameters listed in column 2 in tables 2 and 3.

\section{Estimation of characteristic parameters}

For a demonstration of the proposed algorithm, figure 1 is numerically tested in this section.

\subsection{Case A: Operating speeds in between the second and third critical frequency}

The signals are generated at randomly picked running speeds in between the second $(10.66 \mathrm{~Hz})$ and third $(31.84 \mathrm{~Hz})$ critical frequency. The running speeds picked are $16 \mathrm{~Hz}, 17 \mathrm{~Hz}, 18 \mathrm{~Hz}, 19 \mathrm{~Hz}, 20 \mathrm{~Hz}, 21 \mathrm{~Hz}, 22 \mathrm{~Hz}$, 


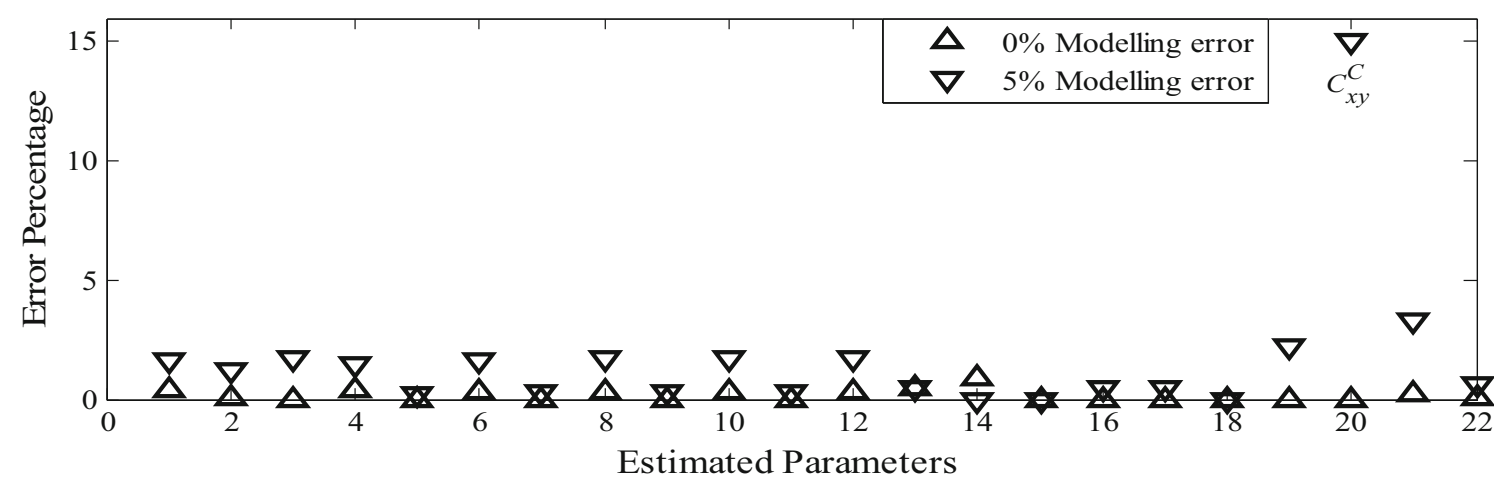

Figure 11. Effect of modelling error on evaluated parameters for Case A.

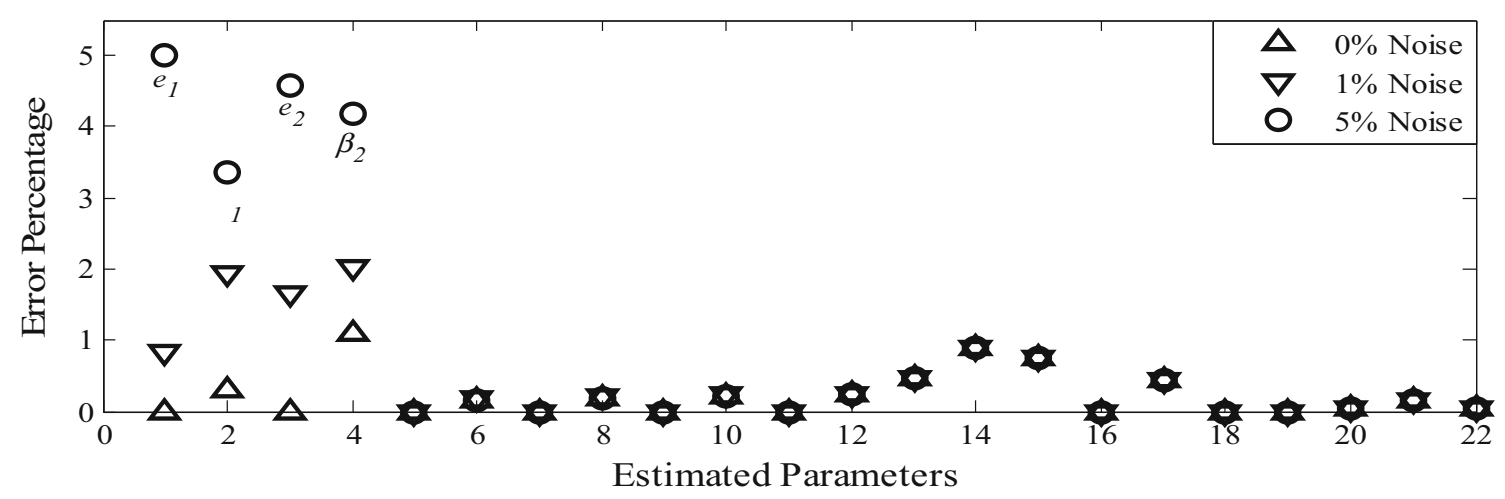

Figure 12. Effect of measurement noise on evaluated parameters for Case B.

$23 \mathrm{~Hz}, 24 \mathrm{~Hz}$ and $25 \mathrm{~Hz}$. The estimated characteristic parameters for various measurement noise levels $(0,1$ and $5 \%)$ are presented in table 2 . To mimic the actual test response noise is deliberately added to the simulated response (time domain signal). The variation on estimated parameters against measurement noise is presented in table 2. From table 2, good agreement could be predicted of evaluated parameters with assumed values. The maximum percentage error in the estimation is around $5.4 \%$ of unbalance parameter. The variations in percentage error for all the estimated parameters are shown in figure 10 . From figure 10, it could be perceived that the AMB and coupling characteristic parameters exhibit very small variation with the addition of measurement noise, whereas the unbalance parameters vary a little as the measurement noise increases up to $5 \%$. The effect of modelling error (5\% variation in mass of the disc and bearing characteristic parameters) on evaluated parameters is carried out and presented in figure 11. From figure 11, the good reliability of the algorithm against modelling error could be observed $\left(C_{x y}^{C}=18.21 \%\right)$. From table 2 , it is evident that absolute assumed value of cross-coupled coupling parameters $\left(C_{x y}\right.$ and $\left.C_{y x}\right)$ are very small as compared with the direct coupling parameters $\left(C_{x x}\right.$ and $\left.C_{y y}\right)$. Since the absolute value is very small, hence, slight deviation in the estimation represents comparatively large percentage error.

\subsection{Case B: Operating speeds in between the fourth and fifth critical frequency}

The signals are generated at randomly picked running speeds in between the fourth $(33.12 \mathrm{~Hz})$ and fifth $(55.25 \mathrm{~Hz})$ critical frequency. The running speeds picked up are $38 \mathrm{~Hz}, 39 \mathrm{~Hz}, 41 \mathrm{~Hz}, 42 \mathrm{~Hz}, 43 \mathrm{~Hz}, 45 \mathrm{~Hz}, 47 \mathrm{~Hz}$, $48 \mathrm{~Hz}, 49 \mathrm{~Hz}$ and $51 \mathrm{~Hz}$. The estimated characteristic parameters for a range of measurement noise $(0-5 \%)$ are tabulated in table 3 . From table 3, well agreement could be predicted of evaluated parameters with assumed values. The maximum percentage error took place for the case is around $4.58 \%$ for unbalance. The variation in percentage error for all the estimated parameters is shown in figure 12 . From figure 12, it could be observed that the dynamic AMB and coupling parameters are steady and very small deviation with the addition of measurement noise took place whereas, the inherent unbalance parameters are varying with the addition of measurement noise. Figure 13 


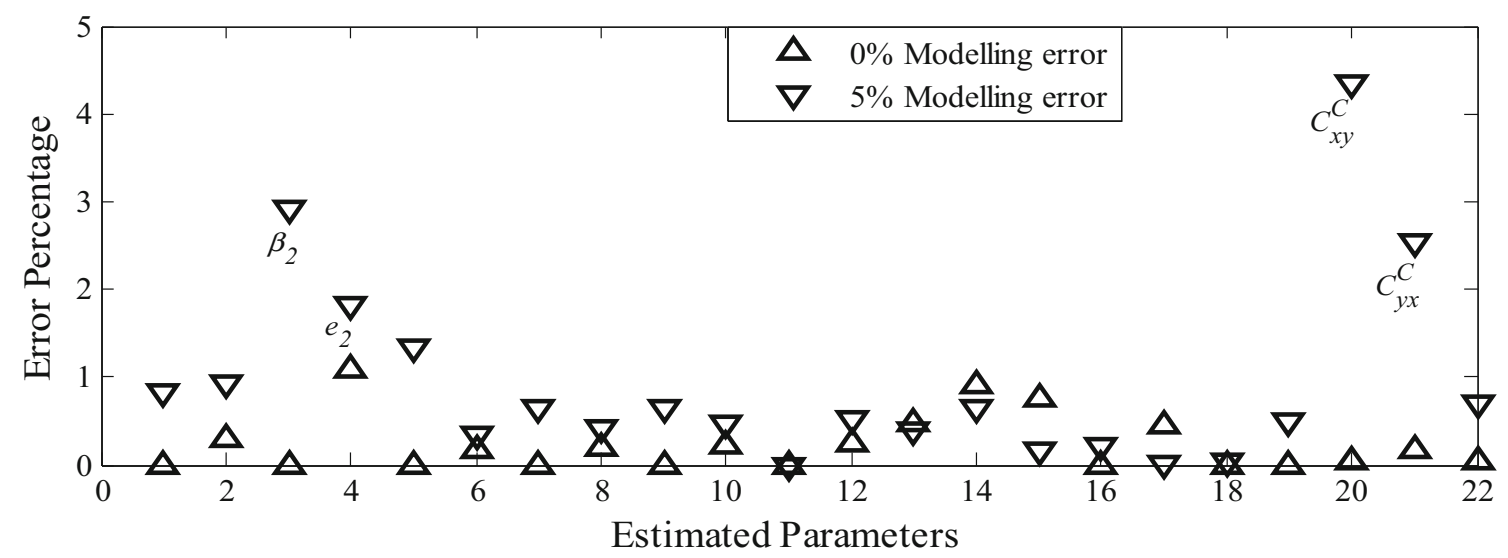

Figure 13. Effect of modelling error on evaluated parameters for Case B.

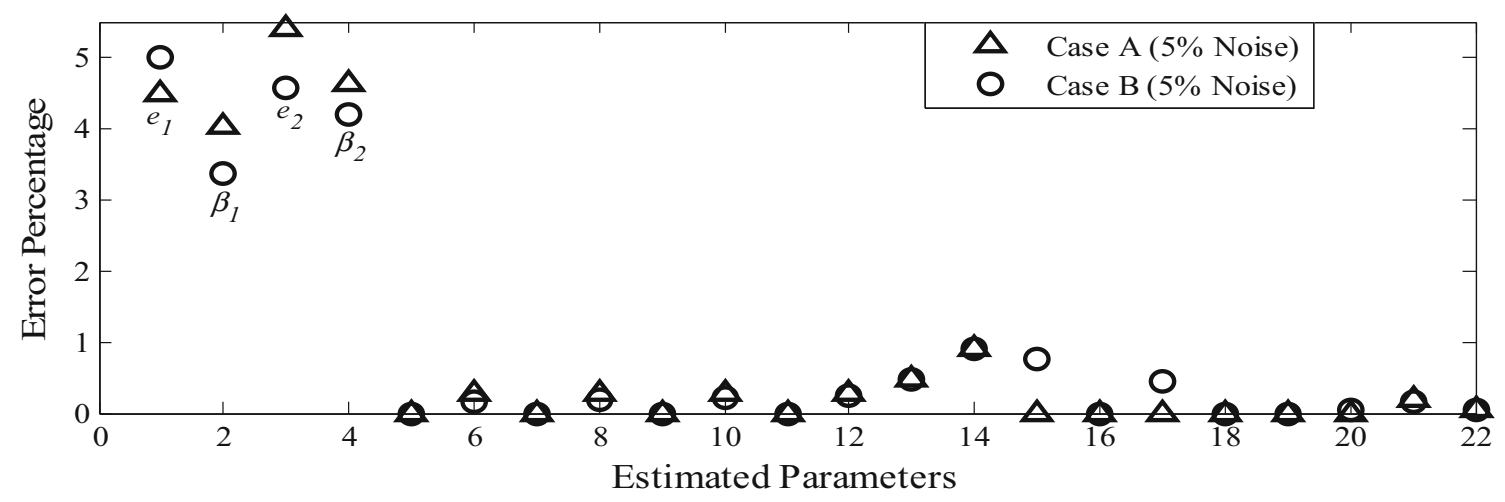

Figure 14. Repeatability analysis of estimated parameters for different cases.

represents the modelling error. Most of the parameters are consistent with the deviation in model parameters. The maximum percentage error occurred due to modelling error is $\left(C_{x y}^{C}=2.21 \%\right)$.

From figures 11 and 13, it can be seen that most of the estimated parameters represent well agreement with the assumed values except the cross-coupled coupling parameter $\left(C_{x y}\right)$ against modelling error. So it can be concluded that the identification algorithm represents well agreement against modelling error, also. From figures 10 and 14 and tables 2 and 3 , it could be concluded that the algorithm exhibit consistent performance with respect to different frequency ranges or different modes of excitation. For completeness, the repeatability of estimated parameters for $5 \%$ measurement noise condition is shown in figure 14 . From figure 14, good resemblance in evaluated parameters may be observed for two different cases considered namely, Case $\boldsymbol{A}$ and Case $B$.

After estimating the system characteristic parameters misalignment forces and moments are calculated and presented in figure 15. The expression for misalignment forces and moments are presented in ref. [28]. The frequency range considered here is same as used for estimating characteristic parameters, i.e., Case A and B (10-50 Hz). From figure 15, an appreciable reduction in the vibration amplitude can be observed by applying the AMB. It is also an indication of a stable system at higher operating modes.

\subsection{Advantages of the proposed method}

The proposed method has the following advantages over other published literature in the past.

a. The excessive vibration generated due to coupling misalignment fault and unbalance is suppressed with the help of active magnetic bearings (AMBs).

b. Tuned AMB characteristic parameters have been estimated along with coupling misalignment and unbalance parameters.

c. High-frequency reduction scheme is applied on AMBrotor-coupling system. 

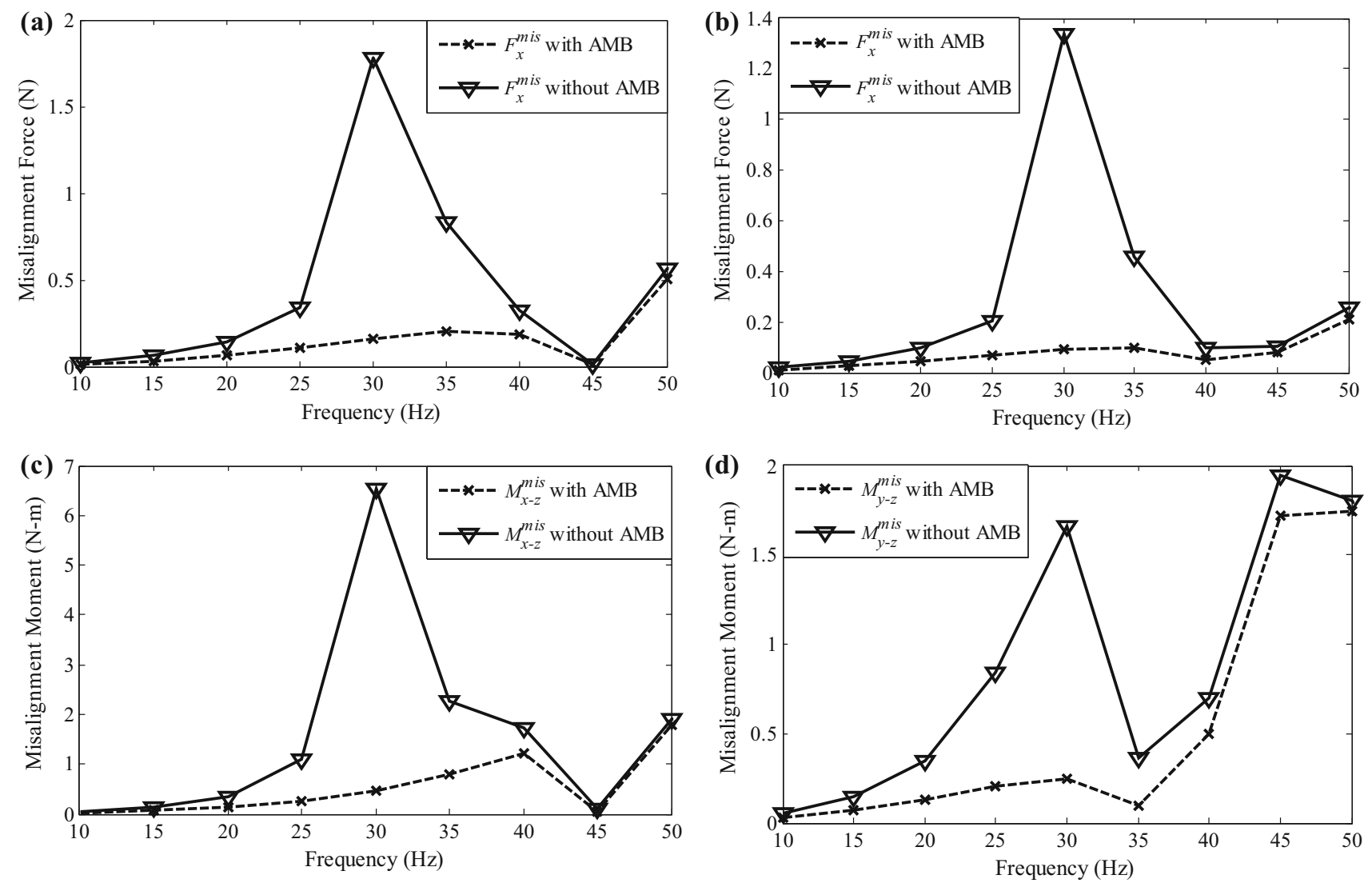

Figure 15. Coupling misalignment forces and moments (with and without AMB) with respect to running frequencies.

d. The proposed algorithm has the flexibility to incorporate any number of discs, shafts, couplings, AMBs and bearings in the system.

\section{Conclusions}

Present research article deals with the development of an identification methodology to evaluate the tuned AMB characteristic parameters for online condition monitoring of the turbogenerator systems represented as coupled flexible rotor system. A known beam theory (Euler Bernoulli) and FEM is utilized to describe the flexible shafts and to obtain elemental equations, respectively. A highfrequency condensation scheme is applied to remove all the rotational DOFs from equations of motion. Also, to reflect the effect of backward and forward whirl, the concept of full spectrum is utilized to quantify the parameters. The novelty of the present work is a simultaneous evaluation of tuned AMB parameters along with inherent unbalance and dynamic coupling parameters. To check the authenticity and adaptability of the developed algorithm, fault parameters are evaluated in the different frequency range (second to fourth natural frequency). An appreciable matching in estimated parameters is observed in the different frequency range. Also, the robustness of the methodology is verified against a range of measurement and modelling error and found compatible with them. The modelling error is simulated by adding percentage deviations in the model parameters i.e., 5\% deviations in disc mass and bearing stiffness. The real challenge will be to validate the algorithm experimentally in the future.

\section{Appendix A}

The peak $(f r+1,1$ and $-f r)$ acquired from figure 9, are utilized to develop the algorithm as,

$$
\boldsymbol{A}\left(\omega_{i}\right) \chi=\boldsymbol{B}\left(\omega_{i}\right)
$$

with,

$$
\boldsymbol{A}\left(\omega_{i}\right)=\left[\boldsymbol{A}_{\boldsymbol{U}}\left(\omega_{i}\right) \boldsymbol{A}_{\boldsymbol{A M B}}\left(\omega_{i}\right) \boldsymbol{A}_{\boldsymbol{C}}\left(\omega_{i}\right)\right]
$$

Regression matrix due to residual unbalance $\boldsymbol{A}_{\boldsymbol{U}}\left(\omega_{i}\right)$ 


$$
\boldsymbol{A}_{\boldsymbol{U}}\left(\omega_{i}\right)=\left[\begin{array}{cccc}
-\boldsymbol{m}^{\boldsymbol{d}_{1}} \omega^{2} \lambda_{\boldsymbol{U}_{1}} & 0 & -\boldsymbol{m}^{\boldsymbol{d}_{2}} \omega^{2} \lambda_{\boldsymbol{U}_{2}} & 0 \\
\vdots & \vdots & \vdots & \vdots \\
0 & -\boldsymbol{m}^{d_{1}} \omega^{2} \lambda_{U_{1}} & 0 & -\boldsymbol{m}^{d_{2}} \omega^{2} \lambda_{U_{2}} \\
\vdots & \vdots & \vdots & \vdots
\end{array}\right]
$$

Regression matrix due to active magnetic bearing $A_{A M B}\left(\omega_{i}\right)$

$$
\boldsymbol{A}_{\boldsymbol{A M B}}\left(\omega_{i}\right)=\left[\begin{array}{ll}
\boldsymbol{A}_{\boldsymbol{A M B} \boldsymbol{B}_{x}} & \boldsymbol{A}_{\boldsymbol{A} M B_{y}}
\end{array}\right]
$$

Regression matrix due to coupling stiffness and damping $A_{C}\left(\omega_{i}\right)$

$$
A_{C}\left(\omega_{i}\right)=\left[A_{C_{k}}\left(\omega_{i}\right) \quad A_{C_{c}}\left(\omega_{i}\right)\right]
$$

$$
\begin{aligned}
A_{A M B_{x}} & =\left[\begin{array}{cccc}
r e\left(\bar{X}_{i}^{\circ} \lambda_{A M B_{x 1}}\right) & r e\left(\bar{I}_{i} \lambda_{A M B_{x 1}}\right) & r e\left(\bar{X}_{i}^{\circ} \lambda_{A M B_{x 2}}\right) & r e\left(\bar{X}_{i} \lambda_{A M B_{x 2}}\right) \\
\vdots & \vdots & \vdots & \vdots \\
0 & 0 & 0 & 0 \\
\vdots & \vdots & \vdots & \vdots
\end{array}\right] \\
A_{A M B_{y}} & =\left[\begin{array}{cccc}
0 & 0 & 0 & 0 \\
\vdots & \vdots & \vdots & \vdots \\
i m\left(\bar{X}_{i}^{\circ} \lambda_{A M B_{y 1}}\right) & i m\left(\bar{I}_{i} \lambda_{A M B_{y 1}}\right) & i m\left(\bar{X}_{i}^{\circ} \lambda_{A M B_{y 2}}\right) & i m\left(\bar{X}_{i} \lambda_{A M B_{y 2}}\right) \\
\vdots & \vdots & \vdots & \vdots
\end{array}\right]
\end{aligned}
$$

$$
\begin{aligned}
& A_{c_{x}}=\left[\begin{array}{cccccc}
r e\left(\bar{X}_{i} \lambda_{x x}^{c_{k}}\right) & \operatorname{re}\left(\bar{X}_{i} \lambda_{x y}^{c_{k}}\right) & \operatorname{re}\left(\bar{X}_{i} \lambda_{y x}^{c_{k}}\right) & \operatorname{re}\left(\bar{X}_{i} \lambda_{y y}^{c_{k}}\right) & \operatorname{re}\left(\bar{X}_{i} \lambda_{\varphi_{x} \varphi_{x}}^{c_{k}}\right) & \operatorname{re}\left(\bar{X}_{i} \lambda_{\varphi_{y} \varphi_{y}}^{c_{k}}\right) \\
\vdots & \vdots & \vdots & \vdots & \vdots & \vdots \\
\operatorname{im}\left(\bar{X}_{i} \lambda_{x x}^{c_{k}}\right) & \operatorname{im}\left(\bar{X}_{i} \lambda_{x x}^{c_{k}}\right) & \operatorname{im}\left(\bar{X}_{i} \lambda_{y x}^{c_{k}}\right) & \operatorname{im}\left(\bar{X}_{i} \lambda_{y y}^{c_{k}}\right) & \operatorname{im}\left(\bar{X}_{i} \lambda_{\varphi_{x} \varphi_{x}}^{c_{k}}\right) & \operatorname{im}\left(\bar{X}_{i} \lambda_{\varphi_{y} \varphi_{y}}\right) \\
\vdots & \vdots & \vdots & \vdots & \vdots & \vdots
\end{array}\right] \\
& A_{C_{c}}=\left[\begin{array}{cccc}
i m\left(\bar{X}_{i} \lambda_{x x}^{c_{c}}\right) & \operatorname{im}\left(\bar{X}_{i} \lambda_{x y}^{c_{c}}\right) & \operatorname{im}\left(\bar{X}_{i} \lambda_{y x}^{c_{c}}\right) & \operatorname{im}\left(\bar{X}_{i} \lambda_{y x}^{c_{c}}\right) \\
\vdots & \vdots & \vdots & \vdots \\
\operatorname{re}\left(\bar{X}_{i} \lambda_{x x}^{c}\right) & \operatorname{re}\left(\bar{X}_{i} \lambda_{x y}^{c_{c}}\right) & \operatorname{re}\left(\bar{X}_{i} \lambda_{y x}^{c_{c}}\right) & \operatorname{re}\left(\bar{X}_{i} \lambda_{y y}^{c_{c}}\right) \\
\vdots & \vdots & \vdots & \vdots
\end{array}\right] \\
& B\left(\omega_{i}\right)=\left[\begin{array}{c}
\omega^{2} M^{H(D+S)} r e\left(\bar{X}_{i}\right)-K^{H(B+S)} r e\left(\bar{\eta}_{i}\right)+\omega C^{H(B+S)} i m\left(\bar{\eta}_{i}\right)+\omega^{2} G^{H(D)} i m\left(\bar{\eta}_{i}\right) \\
\vdots \\
\omega^{2} M^{H(D+S)} i m\left(\bar{X}_{i}\right)-K^{H(B+S)} i m\left(\bar{\eta}_{i}\right)+\omega C^{H(B+S)} r e\left(\bar{\eta}_{i}\right)+\omega^{2} G^{H(D)} r e\left(\bar{\eta}_{i}\right) \\
\vdots
\end{array}\right]
\end{aligned}
$$


Here $\bar{X}_{i}$ and $\bar{I}_{i}(i=f r+1,0,-f r)$ are the displacement and current signals in frequency domain. $\lambda_{U_{i}}$, $\lambda_{A M B_{x i}}, \lambda_{A M B_{v i}},{ }^{o} \lambda_{A M B_{x i}},{ }^{o} \lambda_{A M B_{y i}}(i=1, \quad 2) \quad$ and $\lambda_{x x}^{C_{k}}, \lambda_{x y}^{C_{k}}, \lambda_{y x}^{C_{k}}, \lambda_{y y}^{C_{k}}, \lambda_{\varphi_{x} \varphi_{x}}^{C_{k}}, \lambda_{\varphi_{y} \varphi_{y}}^{C_{k}}, \lambda_{x x}^{C_{c}}, \lambda_{x y}^{C_{c}}, \lambda_{y x}^{C_{c}}, \lambda_{y y}^{C_{c}}$ are the flag location corresponding to unbalance, AMB (displacement and current) and coupling stiffness and damping, respectively.

\section{References}

[1] Schweitzer G and Maslen E H 2009 Magnetic bearings: theory, design, and application to rotating machinery. Berlin: Springer

[2] Tiwari R and Chougale A 2014 Identification of bearing dynamic parameters and unbalance states in a flexible rotor system fully levitated on active magnetic bearings. Mechatronics 24: 274-286

[3] Hili M A, Bouaziz S and Haddar M 2017 Stability analysis and dynamic behaviour of a flexible asymmetric rotor supported by active magnetic bearings. J. Theo. Appl. Mech. 55: 751-763

[4] Burrows C R, Sahinkaya M N and Clements S 1989 Active vibration control of flexible rotors: an experimental and theoretical study. Proc. R. Soc. Lond. A: Math. Phys. Eng. Sci. 422: 123-146

[5] Clark D J, Jansen M J and Montague G T 2004 An overview of magnetic bearing technology for gas turbine engines. NASA

[6] Amer Y A and Hegazy U H 2007 Resonance behavior of a rotor-active magnetic bearing with time-varying stiffness. Chaos, Sol. Fractals 34: 1328-1345

[7] Kasarda M E, Marshall J and Prins R 2007 Active magnetic bearing based force measurement using the multi-point technique. Mech. Research Commun. 34: 44-53

[8] Arredondo I, Jugo J and Etxebarria V 2008 Modelling and control of a flexible rotor system with AMB-based sustentation. ISA Trans. 47: 101-112

[9] Bouaziz S, Messaoud N B, Mataar M, Fakhfakh T and Haddar M 2011 A theoretical model for analyzing the dynamic behavior of misaligned rotor with Active Magnetic Bearing. Mechatronics 21: 899-907

[10] Bouaziz S, Messaoud N B, Choley J Y, Maatar M and Haddar M 2013 Transient response of a rotor-AMBs system connected by a flexible mechanical coupling. Mechatronics 23: $573-580$

[11] Chen S Y and Lin F J 2013 Decentralized PID neural network control for five degree-of-freedom active magnetic bearing. Eng. Appl. Artif. Intell. 26: 962-973

[12] Jiang K, Zhu C, Chen L and Qiao X 2015 Multi-DOF rotor model based measurement of stiffness and damping for active magnetic bearing using multi-frequency excitation. Mech. Syst. Signal. Proces. 60: 358-374

[13] Jin C, Xu Y, Zhou J and Cheng C 2016 Active magnetic bearing stiffness and damping identification from frequency characteristics of control system. Shock Vibr. 2016

[14] Zhou J, Di L, Cheng C, Xu Y and Lin Z 2016 A rotor unbalance response based approach to the identification of the closed-loop stiffness and damping coefficients of active magnetic bearings. Mech. Syst. Signal Process. 66: 665-678

[15] Chen Q, Liu G and Han B 2017 Unbalance vibration suppression for AMBs system using adaptive notch filter. Mech. Syst. Signal. Process. 93: 136-150

[16] Kuppa S K and Lal M 2017 Characteristic Parameter Estimation of AMB Supported Coupled Rotor System. In: Proc. ASME 2017 Gas Turbine India Conference 7-8 December, Bangalore, India pp. V002T05A010-V002T05A010

[17] Kuppa S K and Lal M 2018 Characteristic Parameters Estimation of Uncertainties Present in an Active Magnetic Bearing Integrated Flexible Rotor System Using Dynamic Reduction Technique. In: Inter. Conf. Rotor Dynamics, Springer Cham. 63: 234-248

[18] Spangler D, Prins R and Kasarda M, 2017 A System Identification Technique Using Bias Current Perturbation for the Determination of the Magnetic Axes of an Active Magnetic Bearing. In Actuators. Multidisciplinary Digital Publishing Institute. 6: 13

[19] Xu Y, Zhou J, Di L and Zhao C 2017 Active magnetic bearings dynamic parameters identification from experimental rotor unbalance response. Mech. Syst. Signal. Process. 83: 228-240

[20] Bonfitto A, Castellanos Molina L, Tonoli A and Amati N 2018 Offset-Free Model Predictive Control for Active Magnetic Bearing Systems. In Actuators Multidisciplinary Digital Publishing Institute. 7: 46

[21] Ebrahimi R, Ghayour M and Khanlo H M 2018 Nonlinear dynamic analysis and experimental verification of a magnetically supported flexible rotor system with auxiliary bearings. Mech. Mach. Theory 121: 545-562

[22] Li Q, Wang W, Weaver B and Shao X 2018 Active rotor dynamic stability control by use of a combined active magnetic bearing and hole pattern seal component for back-toback centrifugal compressors. Mech. Mach. Theory 127: $1-12$

[23] Lusty C, Bailey N Y and Keogh P S 2018 Control of Flexible Rotor Vibration with Flexibly Mounted Active Magnetic Bearings. In: Inter. Conf. Rotor Dynamics, Springer, Cham. 62: $65-73$

[24] Lyu M, Liu T, Wang Z, Yan S, Jia X and Wang Y 2018 A control method of the rotor re-levitation for different orbit responses during touchdowns in active magnetic bearings. Mech. Syst. Signal. Process. 105: 241-260

[25] Xu Y, Zhou J, Lin Z and Jin C 2018 Identification of dynamic parameters of active magnetic bearings in a flexible rotor system considering residual unbalances. Mechatronics 49: 46-55

[26] Lal M and Tiwari R 2012 Multi-fault identification in simple rotor-bearing-coupling systems based on forced response measurements. Mech. Mach. Theory 51: 87-109

[27] Rao J S 2011 History of rotating machinery dynamics. Springer Science and Business Media 20

[28] Lal M and Tiwari R 2013 Quantification of Multiple Fault Parameters in Flexible Turbo-Generator Systems with Incomplete Rundown Data. Mech. Syst. Signal. Process. 41: $546-563$

[29] Millsaps K T and Vejvoda C E 1996 Origin of split resonance and backward whirl in a simple rotor. In: ASME 1996 Inter. Gas Turbine and Aeroengine Congress and Exhibition, pp. V003T07A002-V003T07A002 
[30] Lal M and Tiwari R 2018 Experimental identification of shaft misalignment in a turbo-generator system. Sadhana (Acad. Proc. Eng. Sci.) 43: 80

[31] Tiwari R 2017 Rotor systems: analysis and identification. CRC Press, Boca Raton
[32] Paz M 1984 Dynamic condensation. AIAA J. 22: 724-727

[33] Friswell M and Mottershead J E 2013 Finite element model updating in structural dynamics. Springer Science and Business Media 38 